\title{
Spatially resolved star formation and fuelling in galaxy interactions
}

\author{
Jorge Moreno ${ }^{\circledR},{ }^{\star}$ Paul Torrey ${ }^{\circledR}, 2$ Sara L. Ellison ${ }^{\circledR},{ }^{3}$ David R. Patton ${ }^{\circledR},{ }^{4}$ Connor Bottrell ${ }^{\circledR}, 3$ \\ Asa F. L. Bluck ${ }^{\oplus, 5,6}$ Maan H. Hani ${ }^{\oplus},{ }^{3} \dagger$ Christopher C. Hayward $^{\oplus}, 7$ James S. Bullock ${ }^{\oplus}, 8$ \\ Philip F. Hopkins ${ }^{\oplus 9}$ and Lars Hernquist ${ }^{10}$ \\ ${ }^{1}$ Department of Physics and Astronomy, Pomona College, Claremont, CA 91711, USA \\ ${ }^{2}$ Department of Astronomy, University of Florida, 211 Bryant Space Sciences Center, Gainesville, FL 32611, USA \\ ${ }^{3}$ Department of Physics \& Astronomy, University of Victoria, Finnerty Road, Victoria, BC V8P 1A1, Canada \\ ${ }^{4}$ Department of Physics \& Astronomy, Trent University, 1600 West Bank Drive, Peterborough, ON K9L 0G2, Canada \\ ${ }^{5}$ Kavli Institute for Cosmology \& Cavendish Astrophysics, University of Cambridge, Madingley Road, Cambridge CB3 OHA, UK \\ ${ }^{6}$ Hughes Hall College, University of Cambridge, Wollaston Road, Cambridge CB1 2EW, UK \\ ${ }^{7}$ Center for Computational Astrophysics, Flatiron Institute, 162 Fifth Avenue, New York, NY 10010, USA \\ ${ }^{8}$ Department of Physics and Astronomy, University of California, Irvine, CA 92697, USA \\ ${ }^{9}$ TAPIR, California Institute of Technology, Mailcode 350-17, Pasadena, CA 91125, USA \\ ${ }^{10}$ Harvard-Smithsonian Center for Astrophysics, 60 Garden Street, Cambridge, MA 02138, USA
}

Accepted 2020 September 22. Received 2020 September 22; in original form 2020 July 24

\begin{abstract}
We investigate the spatial structure and evolution of star formation and the interstellar medium (ISM) in interacting galaxies. We use an extensive suite of parsec-scale galaxy-merger simulations (stellar mass ratio $=2.5: 1$ ), which employs the 'Feedback In Realistic Environments-2' model (FIRE-2). This framework resolves star formation, feedback processes, and the multiphase structure of the ISM. We focus on the galaxy-pair stages of interaction. We find that close encounters substantially augment cool $(\mathrm{HI})$ and cold-dense $\left(\mathrm{H}_{2}\right)$ gas budgets, elevating the formation of new stars as a result. This enhancement is centrally concentrated for the secondary galaxy, and more radially extended for the primary. This behaviour is weakly dependent on orbital geometry. We also find that galaxies with elevated global star formation rate (SFR) experience intense nuclear SFR enhancement, driven by high levels of either star formation efficiency (SFE) or available cold-dense gas fuel. Galaxies with suppressed global SFR also contain a nuclear cold-dense gas reservoir, but low SFE levels diminish SFR in the central region. Concretely, in the majority of cases, SFR enhancement in the central kiloparsec is fuel-driven (55 per cent for the secondary, 71 per cent for the primary) while central SFR suppression is efficiency-driven ( 91 per cent for the secondary, 97 per cent for the primary). Our numerical predictions underscore the need of substantially larger, and/or merger-dedicated, spatially resolved galaxy surveys - capable of examining vast and diverse samples of interacting systems - coupled with multiwavelength campaigns aimed to capture their internal ISM structure.
\end{abstract}

Key words: methods: numerical-ISM: structure-galaxies: evolution-galaxies: interactions-galaxies: starburst-galaxies: star formation.

\section{INTRODUCTION}

It has been $80 \mathrm{yr}$ since the publication of very first observational and 'numerical' investigations on the nature of galaxy encounters (Holmberg 1940, 1941). Decades later, the emergence of computers allowed researchers to conduct the first numerical experiments of idealized (non-cosmological) galaxy-merging systems (Toomre \& Toomre 1972; Hernquist 1989; Barnes \& Hernquist 1991, 1996; Mihos \& Hernquist 1996) - which supplied a theoretical framework to explain tidally distorted galaxies (Arp 1966; Larson \& Tinsley 1978), and a possible connection between galaxy mergers, starbursts, and quasars (Sanders et al. 1988; Sanders \& Mirabel 1996; Canalizo \& Stockton 2001).

\footnotetext{
${ }^{\star}$ E-mail: jorge.moreno@pomona.edu

$\dagger$ Vanier Fellow.
}

Contemporaneously, galaxy mergers were recognized as naturally occurring events within the hierarchical Lambda cold dark matter ( $\mathrm{CDDM}$ ) paradigm (White \& Rees 1978; Blumenthal et al. 1984; White \& Frenk 1991) - and now form a crucial ingredient in semianalytical models (SAMs) of galaxy formation (Cole et al. 2000; Bower et al. 2006; Croton et al. 2006; De Lucia \& Blaizot 2007; Henriques et al. 2011; Benson 2012; Guo et al. 2013; Lagos et al. 2018, 2019). Often, these SAMs rely on idealized galaxy-merger simulations for guidance. For instance, Hopkins et al. (2008) and Somerville et al. (2008) directly implement results from idealized galaxy-merger simulations by Hopkins et al. (2005) in their cosmological recipes.

Unfortunately, the great majority of SAMs entirely ignore the early stages of interaction - i.e. when the merging galaxies can still be identified as two distinct units (but see Menci et al. 2004, for an exception). This is despite the fact that a vast number of 
observations reveal their definitive importance. Concretely, in the local Universe, interaction-induced star formation is enhanced in galaxies with close companions (Patton et al. 1997; Barton, Geller \& Kenyon 2000; Lambas et al. 2003; Ellison et al. 2008; Scudder et al. 2012; Robotham et al. 2014). Close galaxy encounters also diminish nuclear metallicity (Rupke, Kewley \& Chien 2010; Rich et al. 2012; Scudder et al. 2012), augment molecular gas content (Violino et al. 2018), mould the circumgalactic medium (Hani et al. 2018; Smith et al. 2018, 2019), and ignite active galactic nuclei (AGNs; Ellison et al. 2011; Treister et al. 2012; Sabater, Best \& Argudo-Fernández 2013; Satyapal et al. 2014; Ellison et al. 2019). Idealized simulations confirm these effects during the pre-merger 'galaxy-pair' period: including enhanced star formation (Di Matteo et al. 2007, 2008; Moreno et al. 2015), decrements in nuclear metallicity (Torrey et al. 2012), alterations in the structure of interstellar medium (ISM; Moreno et al. 2019), and triggered AGN (Di Matteo, Springel \& Hernquist 2005; Callegari et al. 2009; Capelo et al. 2015, 2017).

Unlike the dramatic, albeit brief, turmoil experienced by merging galaxies at coalescence, the effects sparked by the influence of a close neighbour during the early stages of interaction tend to be gentler and of longer duration (Moreno et al. 2015). Observations by Patton et al. (2013) suggest that interaction-driven effects extend out to $\sim 150 \mathrm{kpc}$ in projected separation. Patton et al. (2020) confirm this effect in cosmological simulations, and demonstrate that close encounters affect galaxy pairs out to separations of $\sim 280 \mathrm{kpc}$ in 3D space. Each interaction and flyby (Moreno 2012; Sinha \& HolleyBockelmann 2012; L'Huillier, Park \& Kim 2015; An et al. 2019) is capable of inciting bar formation (Lokas et al. 2016; Pettitt \& Wadsley 2018; Łokas 2019; Cavanagh \& Bekki 2020) and promoting bulge mass growth (Just et al. 2010; Bekki \& Couch 2011). But more importantly, the cumulative effect of multiple - frequently occurring and long-lived - galaxy encounters may ultimately stimulate the transformation of spirals into lenticulars in dense environments (Moore et al. 1996; Boselli \& Gavazzi 2006; Cappellari 2013; Joshi et al. 2020). In sum, galaxy pairs offer a unique and powerful window to understand how close encounters affect both global properties and the internal structure of galaxies.

Coincidentally, recent years have also witnessed the emergence of integral-field unit (IFU) surveys targeting the local Universe such as the Calar Alto Legacy Integral Field Area Survey (CALIFA; Sánchez et al. 2012), the Sydney-AAO Multi-object Integral field spectrograph Galaxy Survey (SAMI; Croom et al. 2012), and the Mapping Nearby Galaxies at APO Survey (MaNGA; Bundy et al. 2015) - which open a new avenue for studying the connection between galactic structure and interaction history. These spatially resolved campaigns allow us to go beyond asking only about global properties - and permit us to analyse the spatial extent of star formation rate (SFR) enhancements (along with the flattening of metallicity gradients; Barrera-Ballesteros et al. 2015a; Pan et al. 2019; Thorp et al. 2019), plus kinematic signatures (Barrera-Ballesteros et al. 2015b; Hung et al. 2016; Bloom et al. 2017; Li et al. 2019; Feng et al. 2020) in interacting galaxies. Coupled with interferometric follow-up observations focused on $\mathrm{H}_{2}$ content and its structure such as EDGE-CALIFA ${ }^{1}$ (Bolatto et al. 2017) and ALMaQUEST ${ }^{2}$ (Lin et al. 2019), we now have the potential to clearly define the

spatial extent in which galaxy interactions affect the ISM, and how this process fuels star formation.

On the numerical side, it is understandable to ask whether idealized (non-cosmological) galaxy-merger simulations, like the ones we present in this paper, are the optimal tool for these kind of studies. Indeed, cosmological simulations (e.g. Perez et al. 2006; Perez, Michel-Dansac \& Tissera 2011; Bustamante et al. 2018; Blumenthal et al. 2020; Hani et al. 2020b; Patton et al. 2020) and zoom-in simulations (e.g. Sparre \& Springel 2016) also provide a viable path. Furthermore, such simulations naturally provide the diversity and cosmological context experienced by merging galaxies in the real Universe (Martig \& Bournaud 2008; Moreno et al. 2013). However, unlike cosmologically selected galaxy pairs, idealized simulations offer unparalleled spatial and temporal resolution. Moreover, this non-cosmological framework renders the user control to conduct numerical experiments where specific initial orbital conditions can be designed to answer specific questions - e.g. the effect of spinorbit inclination and impact parameter, to name a few. In principle, one can also address these questions with cosmological simulations. Unfortunately, when those simulations include hydrodynamics and explicit stellar feedback, their box sizes tend to be small - which may severely limit the diversity in merging orbits. Maximizing high-resolution and sizable volumes remains a challenge for such large-scale simulations - although the use of 'genetically modified' zoom-in technology offers promise to overcome this limitation (Roth, Pontzen \& Peiris 2016; Rey \& Pontzen 2018; Stopyra et al. 2020).

Similarly, in the idealized galaxy-merger approach, expanding diversity in orbital geometries also conspires against increments in resolution. Merger libraries capable of broadly exploring orbital parameter space (e.g. Di Matteo et al. 2008; Moreno et al. 2015) are often forced to employ low-resolution schemes to optimize computational resources. Increases in resolution must also be accompanied with physically motivated sub-grid recipes capable of capturing the multiphase structure of the ISM at those scales. Conversely, when high-resolution programmes (with improved physics prescriptions) attempt to simulate galaxy mergers at the parsec scale, they do so at the expense of not being able to explore orbital parameter space in great detail (Kim, Wise \& Abel 2009; Renaud et al. 2009, 2014, 2019a,b; Karl et al. 2010, 2013; Teyssier, Chapon \& Bournaud 2010; Karl, Fall \& Naab 2011; Hopkins et al. 2013a; Renaud, Bournaud \& Duc 2015).

In this paper, we employ an extensive suite of parsec-scale galaxymerger simulations (Moreno et al. 2019) based on the 'Feedback In Realistic Environments-2' (FIRE-2) model (Hopkins et al. 2018). This framework captures the multiphase structure of the ISM and resolves the physics of relevant feedback processes that regulate star formation. Our suite consists of 24 orbital configurations, making it the largest library of galaxy-merger simulations at the parsec scale to date. This uniquely positions us to conduct spatially resolved studies of star formation and the evolution of the ISM in interacting galaxies from a numerical perspective. This work expands on Moreno et al. (2019), who only address interaction-induced effects on the ISM for the entire two-galaxy system, and Moreno et al. (2015), who analyse the spatial extent of interaction-induced SFR using an older model. With this in mind, this paper addresses the following questions:

\footnotetext{
${ }^{1}$ EDGE stands for the Extragalactic Database for Galaxy Evolution Survey.

${ }^{2}$ ALMaQUEST stands for ALMA-MaNGA QUEnching and STar formation Survey, while ALMA stands for Atacama Large Millimeter/submillimeter Array.
}

(1) How are the new stellar and ISM budgets - as well as the instantaneous SFR and star formation efficiency (SFE, equation 1) in a galaxy affected by the presence of a companion? 
(2) How do these quantities evolve globally, within the central region, and in the rest of the galaxy?

(3) How do close galaxy encounters affect the radial structure of the above baryonic components?

(4) How does the radial structure of galaxies evolve in time during the interaction?

(5) How does our choice of orbital merging geometry affect the radial structure in these baryonic components?

(6) Is there a connection between global SFR enhancement and the radial structure of SFR, SFE, and fuel availability?

(7) What drives SFR enhancement (or suppression) in the central kiloparsec, SFE or fuel availability?

(8) Do the primary and secondary galaxies exhibit different behaviour during the interaction?

This manuscript is organized as follows. Section 2 introduces our simulations and relevant terminology (boldface italics). Sections 3.1, 3.2 , and 3.3, respectively, focus on time evolution, sample-wide averages, and radial structure. We investigate the role of orbital merging geometry in Section 3.4. In Section 3.5, we address connections between global SFR deviations and radial structure, and in Section 3.6, we focus on what factors drive SFR in the central kiloparsec. Section 4 summarizes our findings.

\section{SIMULATIONS AND TERMINOLOGY}

Our galaxy-merger simulations employ the FIRE-2 physics model. See Hopkins et al. $(2014,2018)$ for details - we only provide a brief summary here.Star formation is constrained to selfgravitating, self-shielding (Krumholz \& Gnedin 2011) gas denser than $1000 \mathrm{~cm}^{-3}$ (Hopkins, Narayanan \& Murray 2013b). Once these conditions are met, gas is converted into stars at 100 per cent efficiency per local dynamical time. We incorporate free-free, photoionization/recombination, Compton, photoelectric, dustcollisional, cosmic ray, molecular, metal-line, and fine-structure processes in our treatment of radiative heating and cooling. Our feedback model includes momentum flux from radiation pressure; energy, momentum, mass, and metal injection from Type Ia and II supernovae ( $\mathrm{SNe}$ ), plus mass-loss from $\mathrm{OB}$ and asymbiotic giant brach stars. We use STARBURST99 (Leitherer et al. 1999) to tabulate stellar masses, ages, metallicities, feedback event rates, luminosities, energies, and mass-loss rates. Our simulations employ the meshless finite mass (MFM) mode of the GIZMO hydro solver (Hopkins 2017). We do not incorporate AGN feedback because (1) we wish to focus on the role of stellar feedback alone, and (2) the coupling of AGN fueling and feedback with the surrounding multiphase ISM at the scales probed in this paper is not yet fully understood (but see e.g. Hopkins et al. 2016; Anglés-Alcázar et al. 2017, 2020, for recent work exploring this question).

Our galaxy-merger suite consists of 24 major mergers (stellar mass ratio $=2.5: 1$ ) with three spin-orbit orientations: nearprograde, near-polar, and near-retrograde (Moreno et al. 2015). Each orientation spans the following separations at first pericentric passage: $\sim 7 \mathrm{kpc}$ (three orbits), $\sim 16 \mathrm{kpc}$ (three orbits), and $\sim 27 \mathrm{kpc}$ (two orbits). Initially, the secondary galaxy has the following properties: stellar mass $=1.2 \times 10^{10} \mathrm{M}_{\odot}$, bulge mass $=7.0 \times 10^{9} \mathrm{M}_{\odot}$, and gas mass $=7.0 \times 10^{10} \mathrm{M}_{\odot}$; and the primary has stellar mass $=3.0 \times 10^{10}$, bulge mass $=2.5 \times 10^{10}$, and gas mass $=8.0 \times 10^{10} \mathrm{M}_{\odot}$. We follow Mendel et al. (2014) and Saintonge et al. (2016) for our bulge and gas mass choices. For comparison, we also simulate these two galaxies in isolation. Time outputs are stored at 5-Myr resolution. We adopt gas and stellar particle masses of $1.4 \times 10^{4}$ and $1.9 \times 10^{3} \mathrm{M}_{\odot}$,
Table 1. Temperature-density demarcations: the colddense and cool ISM regimes, adopted to approximately represent $\mathrm{H}_{2}$ and $\mathrm{H}$ I gas, respectively. See Moreno et al. (2019) for details.

\begin{tabular}{lc}
\hline ISM regime & Temperature-density demarcations \\
\hline Cold-dense & $\left(T<300 \mathrm{~K}, n>10 \mathrm{~cm}^{-3}\right)$ \\
Cool & $\left(T<8000 \mathrm{~K}, 0.1 \mathrm{~cm}^{-3}<n<10 \mathrm{~cm}^{-3}\right)$ \\
& and $(300 \mathrm{~K}<T<8000 \mathrm{~K}, n<$ \\
& $\left.0.1 \mathrm{~cm}^{-3}\right)$ \\
\hline
\end{tabular}

respectively. We achieve a maximum spatial resolution of 1.1 parsec and a maximum gas-density resolution of $5.8 \times 10^{5} \mathrm{~cm}^{-3}$. With these choices, $\sim 0.15$ per cent of the gas exceeds the $1000 \mathrm{~cm}^{-3}$ density threshold required (but not necessarily sufficient) for star formation. See tables 1 and 2 of Moreno et al. (2019) for more details on our adopted initial conditions.

We characterize the ISM in terms of four density-temperature demarcations: hot, warm, cool, and cold-dense regimes - meant to represent gas above $10^{6} \mathrm{~K}$, warm-ionized gas, $\mathrm{H} \mathrm{I}$ gas, and $\mathrm{H}_{2}$ gas (Moreno et al. 2019). In this work, we only discuss the cool and cold-dense gas components (Table 1) because these phases are more closely connected to star formation fuelling. In particular, we adopt the temperature and density thresholds of Orr et al. (2018) for our cold-dense gas regime because ISM at densities above $n>10 \mathrm{~cm}^{-3}$ (at solar luminosity) captures the majority of the $\mathrm{H}_{2}$ gas (Semenov, Kravtsov \& Gnedin 2017).

One can write the instantaneous SFR as

$\mathrm{SFR}=\frac{\mathrm{SFR}}{M_{\text {cold-dense }}} \times M_{\text {cold-dense }} \equiv \mathrm{SFE} \times M_{\text {cold-dense }}$,

where SFE denotes the star formation efficiency. Writing SFR in this particular format facilitates our discussion of how these three quantities are interconnected (Sections 3.1-3.6). Indeed, the ability to probe variations in SFE is one of the advantages of using simulations like FIRE. We note this exercise is not entirely appropriate for older simulations that tune their star formation recipes to the observed KS (Kennicutt 1998) law (e.g. Springel \& Hernquist 2003). However, simulations like ours, capable of capturing the multiphase structure of the ISM - and for which the KS naturally emerges as an output (Orr et al. 2018) - provide an appropriate framework to study variations in SFE.

To gain insight, we devote special attention to a specific fiducial run: a nearly prograde configuration with small impact parameter $(\sim 7 \mathrm{kpc}$ ), selected to maximize the effects of the encounter (Moreno et al. 2019). Fig. 1 illustrates this run at three representative times. The top panel shows galaxy-galaxy separation versus time. For each galaxy, we use the location of the central supermassive black hole $(\mathrm{SMBH})$ as a proxy for galactic centre. We note that, in our simulations, this SMBH does not play a dynamical role, but only serves to trace the potential minimum. The thick vertical lines (from left to right) indicate first and second pericentric passages, plus coalescence. Throughout this paper, time is shifted to zero at first pericentric passage. This work focuses on the galaxy-pair period, corresponding to times between first and second pericentric passage. The grey boxes and thin portions of the galaxy-separation curve indicate times outside the galaxy-pair period. To avoid contamination from the companion galaxy, we exclude times at which two 10-kpc spheres surrounding each galaxy overlap. Without this extra condition, one cannot disentangle dynamical effects driven by a recent encounter from the simple presence of new material 'belonging' to (i.e. being closer to the centre of) the companion galaxy. The thin horizontal grey box and the small extra grey spaces right and left of 


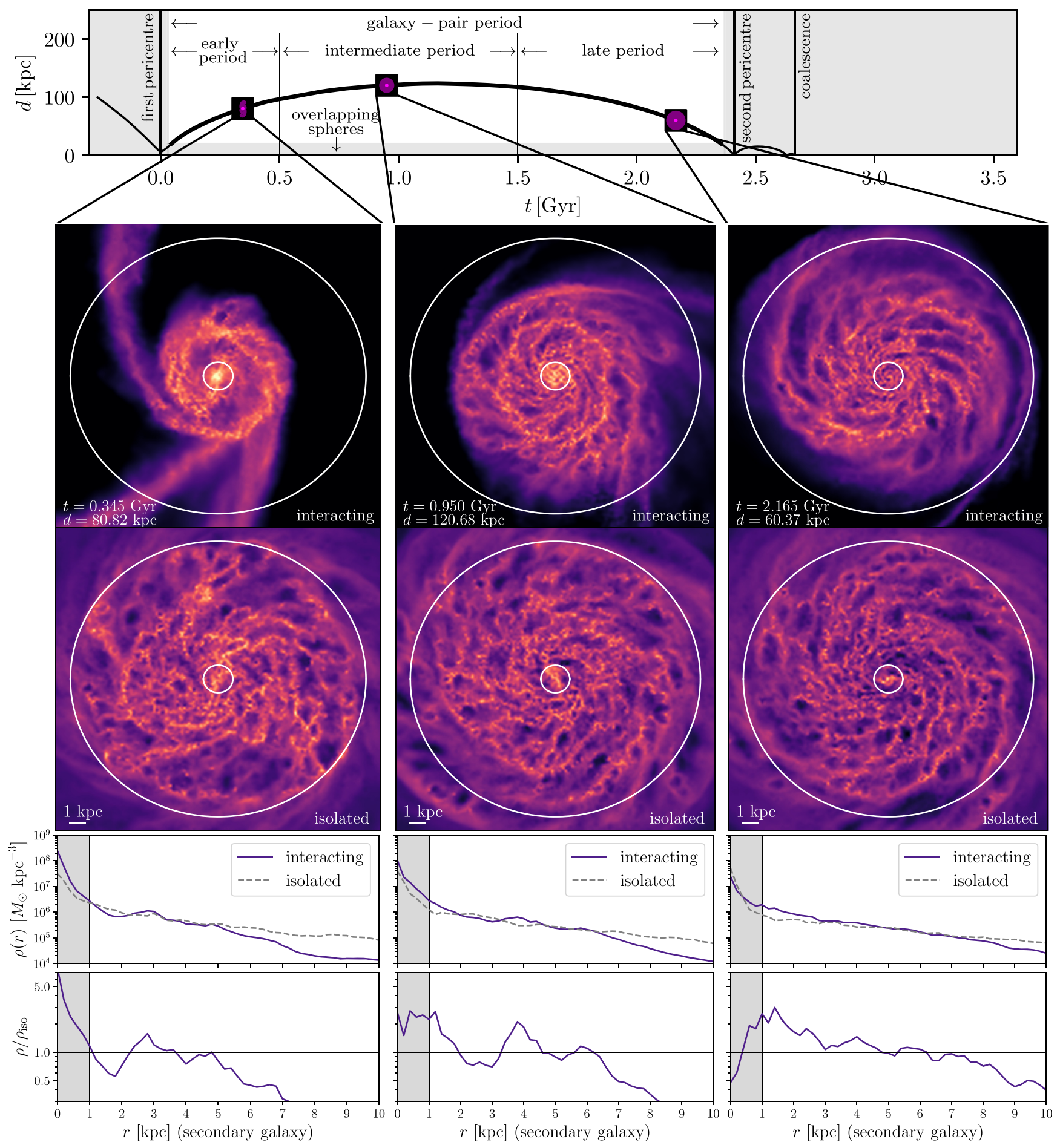

Figure 1. Terminology and three illustrative examples. Top panel: Galaxy-galaxy distance versus time (fiducial run). The thick vertical lines from left to right denote first and second pericentric passages, plus coalescence. Time is shifted to zero at first passage. The thick portion of the curve and the area not covered by grey boxes denote the galaxy-pair period, defined to be between first and second passages, with separations of at least $20 \mathrm{kpc}$. The thin vertical lines indicate the early, intermediate, and late periods, chosen arbitrarily to describe the evolution of the interaction. The solid purple symbols depict three specific times, described further in subsequent panels (increasing in time from left to right). Second (third) row panels: Face-on surface density maps of the entire gas budget - i.e. all ISM phases - for the interacting (isolated) galaxy at three illustrative times, indicated by the three vertical rows. The colour scale is logarithmic (mass weighted) and the same for both rows (all six images). We only show the secondary galaxy and its isolated counterpart. The white circles indicate the centre $(0-1 \mathrm{kpc})$ and the outskirts $(1-10 \mathrm{kpc})$. The galaxy-pair period excludes times when the larger circles belonging to each galaxy overlap. The keys indicate time and galaxy-galaxy distance after first pericentric passage, plus the spatial scale. See supplementary material for videos associated with these images. Fourth (fifth) row panels: 3D radial gas mass density profiles for the interacting (purple) and isolated (dashed grey) galaxy, plus their ratio (purple). The vertical line at $1 \mathrm{kpc}$ and the grey box indicate the central region. The horizontal line indicates unity. 
the first and second thick vertical lines, respectively, highlight this extra condition.

To describe how the spatial extent of various baryonic components evolves in time, we split the galaxy-pair period into three portions: the early $(t<0.5 \mathrm{Gyr})$, intermediate $(0.5<t<1.5 \mathrm{Gyr})$, and late $(t>1.5 \mathrm{Gyr})$ time periods. The two thin vertical lines at $t=0.5$ and 1.5 Gyr separate these three periods (Fig. 1, top panel). We keep these lines in figures displaying time evolution throughout the paper to facilitate cross-reference between Sections 3.1 and 3.3. There is no fundamental reason behind these three specific choices. One possibility is to split the galaxy-pair period according to where the two galaxies are located in their merging orbit (Privon 2014; Pan et al. 2019) -i.e. if they are close to first pericentric passage, apocentre, etc. However, this would require replacing our universal demarcations (at 0.5 and $1.5 \mathrm{Gyr}$ ) with orbit-dependent choices, because the duration of the galaxy-pair period varies from merger to merger. We elect to avoid this approach because the large-scale duration of the interaction does not necessarily dictate the time-scales governing small-scale hydrodynamics and feedback-regulated baryonic physics. In the absence of a rigorous option, we visually inspect the gas component in our fiducial secondary galaxy to guide our prima facie choices. ${ }^{3}$ We focus on the gaseous component because interaction-induced disturbances are more extended and visually evident (Bournaud et al. 2004). We inspect the secondary galaxy in detail here (and typically describe it first throughout the paper - i.e. by placing information pertaining to the primary galaxy on the right or bottom panels in figures) because its shallower gravitational potential makes it more susceptible to the effects of the encounter (Barnes \& Hernquist 1996; Di Matteo et al. 2007; Moreno et al. 2015).

Our visual inspection reveals the following sequence of events. (1) Soon after its first pericentric passage, the gaseous component of the galaxy exhibits extended tidal tails, while its disc shrinks and develops a prominent central concentration. (2) External material originally launched into the bridge and tidal tails is re-accreted on to the outskirts of the disc, which begins to recover in radial extent. (3) Lastly, disturbances produced by the encounter fade away and the central concentration becomes diluted. See e.g. Blumenthal \& Barnes (2018) for a more rigorous description of this process. Our demarcations at 0.5 and $1.5 \mathrm{Gyr}$ approximately mark the time-scales at which these three steps unfold. From left to right, the secondrow panels in Fig. 1 display face-on surface-density maps of the gas component (all ISM phases) at specific representative times selected from each of these three periods. Third-row images show similar maps for its isolated counterpart. All six images share the same logarithmic mass-weighted colour scale. To facilitate our analysis (as in Moreno et al. 2015), the white circles split each of our galaxies into the following spherically symmetric 3D regions: the centre ( $r$ $<1 \mathrm{kpc})$, the outskirts $(1<r<10 \mathrm{kpc})$, and the entire galaxy $(r<$ $10 \mathrm{kpc}$ ) - see also Patton et al. (2013), who use the same definition for their central region.

To quantify the spatial extent of the baryonic content in our simulated galaxies, we employ 3D radial density profiles encompassing distances between 0 and $10 \mathrm{kpc}$ from the centre of each galaxy. The fourth-row panels of Fig. 1 show mass density profiles corresponding to the second- and third-row images directly above them. The vertical line and grey box highlight the centre $(r<1 \mathrm{kpc})$. The solidpurple and dashed grey lines represent the profile corresponding to the interacting and isolated galaxy, respectively. To disentangle

${ }^{3}$ For videos of our galaxy-merger simulations, see the online supplementary material and please visit https://research.pomona.edu/galaxymergers/videos/ the effect of the interactions from secular effects, we calculate the ratio of the two. We show this in the fifth-row panels. The horizontal line represents unity. Values above this line indicate enhancement or excess, while those below indicate suppression or deficit. We informally use the word enhancement to encompass these terms (e.g. sub-unity 'enhancement' means deficit). One must be careful while interpreting the meaning of a profile ratio. For instance, from left to right, the profile ratios in the fifth-row panels plummet at large radii. This does not mean that there is no gas there, but rather, that the gas mass content in the interacting galaxy is orders of magnitude below that of its isolated counterpart (purple versus grey lines in the fourth-row panels, and regions immediately inside the larger white circles in the second-row versus third-row images). Similarly, the profile ratio in the centre evolves from strongly enhanced to suppressed. The central deficit at late times does not necessarily mean that there is a 'hole' in the inner gas distribution - rather, it generally means that the original central peak is diminished relative to the central concentration in the secularly evolving isolated galaxy. Although, occasionally, we do witness brief gas evacuation episodes of the central 100-parsec region, which is then quickly replenished by new gas from the surroundings (Torrey et al. 2017).

It is impractical to analyse every individual radial density profile and profile ratio for 24 galaxy mergers at $5 \mathrm{Myr}$ time resolution, which corresponds to approximately twenty thousand individual profiles per baryonic component in the galaxy-pair period alone! Rather, we employ average density profiles and average profile ratios, which are the result of averaging profiles (or profile ratios) over multiple times and orbital configurations in our merger suite. For the profile ratios, we match the interacting and isolated galaxies to be situated at the same time after the start of the simulation. Fig. 2 illustrates this. We display the mass in new stars here (rather than gas, as in Fig. 1) because explaining the effectiveness of interactioninduced star formation is one of the central goals of this paper (Section 3.4). The secondary galaxy is placed on the left-hand panels, while the primary on the right-hand panels. The diagonally hatched brown bands in the top panels show the average density profiles for galaxies with a companion, while the horizontally hatched grey bands correspond to their isolated counterparts. Band thickness represents one standard deviation. The vertical line and grey box highlight the centre $(r<1 \mathrm{kpc})$. Similarly, the bottom panels show the result of averaging profile ratios. The horizontal line indicates unity. Note that the average profile ratio (hatched band, bottom panels) is not merely the ratio of the average density profiles (brown band divided by grey band, top panels). This is because the average of the ratios is not necessarily equal to the ratio of the averages.

While it is true that the results in Fig. 2 offer interesting average trends, teasing out the interaction-induced effects that govern the spatial distribution and evolution of new stars in galaxies is not trivial and depends on many factors. These include (1) the time of observation after the first encounter; (2) the orbital geometry of the encounter; (3) the amount of fuel available; and (4) the SFE - to name a few. To address the first point, in Section 3.3, we make use of the early, intermediate, and late periods defined above. For the second point, we group our 24 mergers into three subsuites:

(i) The typical subsuite (66.7 per cent, 16/24 mergers, fiducal run included): $\{$ near prograde $\} \cup\{$ near polar with first passage at $\sim 16$ and $\sim 27 \mathrm{kpc}\} \cup\{$ near retrograde with first passage at $\sim 7 \mathrm{kpc}\}$.

(ii) The vigorous subsuite (12.5 per cent, $3 / 24$ mergers): $\{$ near polar with first pericentric passage at $\sim 7 \mathrm{kpc}\}$.

(iii) The gentle subsuite (20.1 per cent, 5/24 mergers): \{near retrograde with first passage at $\sim 16$ and $\sim 27 \mathrm{kpc}\}$. 

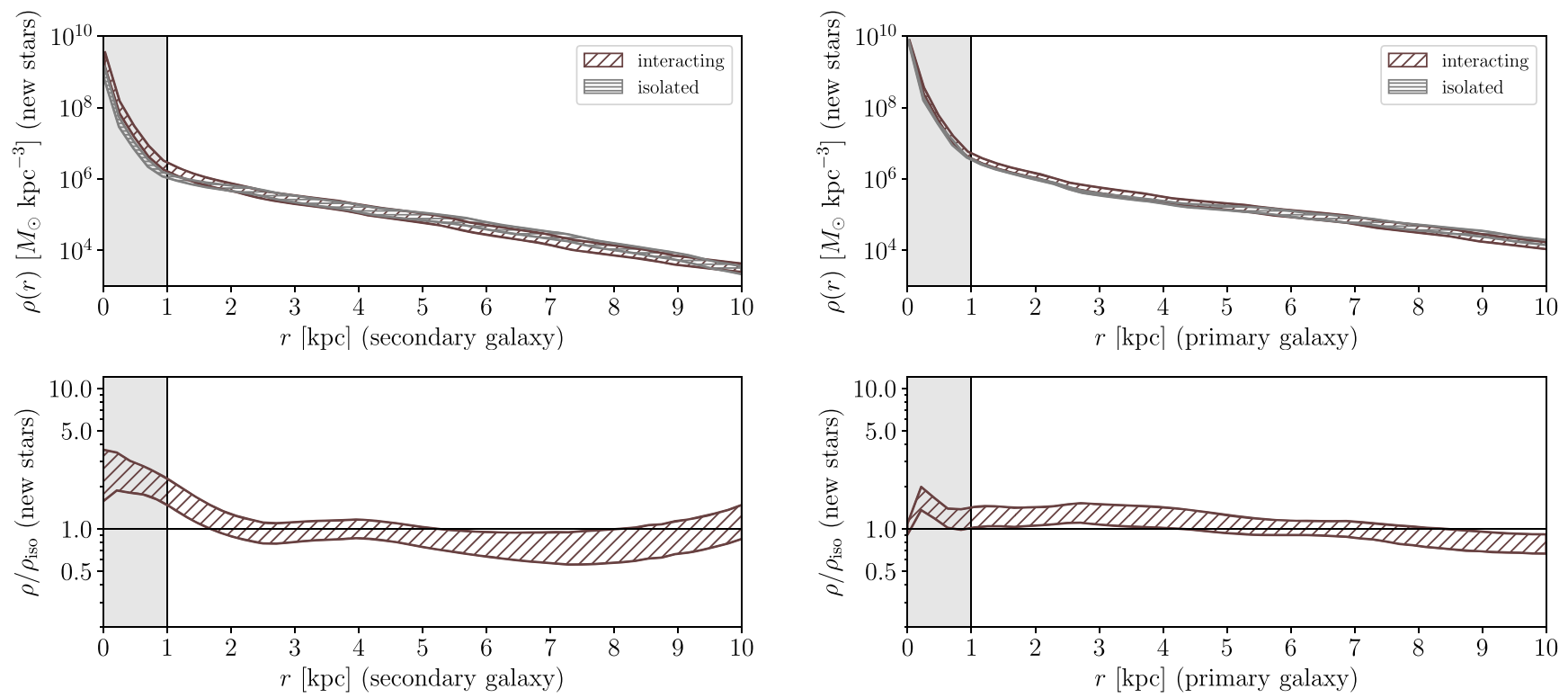

Figure 2. Definitions: average density profile and average profile ratio (mass in new stars, entire merger suite, galaxy-pair periods only). Left (right-hand) panels: secondary (primary) galaxy. Top panels: The diagonally hatched brown band represents the average density profile in new stellar mass versus $3 \mathrm{D}$ radial distance for the interacting galaxy - calculated by averaging across every configuration in our merger suite within their respective galaxy-pair periods. The grey horizontally hatched band is the result of the same exercise for its isolated counterpart. Band thickness represents one standard deviation. Bottom panels: The brown hatched band displays the average profile ratio, which is the result of averaging the ratios of individual density profiles for the interacting galaxy and its isolated counterpart. The vertical black line and grey box highlight the central region $(r<1 \mathrm{kpc})$. The horizontal line indicates unity.

We explain these subsuite-naming conventions in Section 3.4. To address the third and fourth factors mentioned above, we also split the merger suite by global SFR enhancement into three populations, or star-forming $(S F)$ types:

(i) Enhanced star formers (SFs): $\log \mathrm{SFR} / \mathrm{SFR}_{\text {iso }}>+0.3$,

(ii) Regular SFs: $-0.3<\log \mathrm{SFR} / \mathrm{SFR}_{\text {iso }}<0.3$,

(iii) Suppressed SFs: $\log \mathrm{SFR} / \mathrm{SFR}_{\mathrm{iso}}<-0.3$,

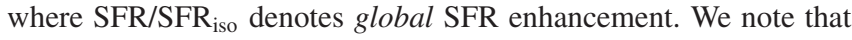
any particular run can 'visit' all three SF-type regimes at different times during the interaction. We justify our global SFR cuts in Section 3.5, where we investigate the connection between location relative to the global $\mathrm{SFR} / \mathrm{SFR}_{\text {iso }}=1$ line and the radial structure of SFR, SFE, and available fuel. Lastly, in Section 3.6, we define

(i) Efficiency-driven enhanced star formation:

$\mathrm{SFE} / \mathrm{SFE}_{\text {iso }}>M_{\text {cold-dense }} / M_{\text {cold-dense, iso, }}$

(ii) Efficiency-driven suppressed star formation:

$\mathrm{SFE} / \mathrm{SFE}_{\text {iso }}<M_{\text {cold-dense }} / M_{\text {cold-dense, iso, }}$,

(iii) Fuel-driven enhanced star formation:

$\mathrm{SFE} / \mathrm{SFE}_{\text {iso }}<M_{\text {cold-dense }} / M_{\text {cold-dense, iso }}$,

(iv) Fuel-driven suppressed star formation:

$\mathrm{SFE} / \mathrm{SFE}_{\text {iso }}>M_{\text {cold-dense }} / M_{\text {cold-dense, iso }}$,

to evaluate the relative contribution of SFE and available cold-dense fuel in driving SFR in the central kiloparsec.

\section{RESULTS AND DISCUSSION}

\subsection{Time evolution}

Before discussing the spatial extent of the new stars and the colddense/cool ISM phases, Fig. 3 addresses the time evolution of our fiducial run in three regions: globally (within $10 \mathrm{kpc}$, mediumcoloured dashed lines), in the centre (within $1 \mathrm{kpc}$, dark solid lines), and in the outskirts (between 1 and $10 \mathrm{kpc}$, light solid lines). We focus on the galaxy-pair period (thick portions of the coloured lines that are not masked by grey boxes). The secondary galaxy (left-hand panels) exhibits the following behaviour. The mass in new stars in the centre (dark brown) is enhanced suddenly and to high levels within the first $\sim 0.3 \mathrm{Gyr}$ after the first encounter, and decreases at later times as stellar-mass growth in the interacting galaxy slows down relative to the isolated one. This growth is driven by the generally strongly enhanced SFR (dark purple) during the galaxy-pair period. We note that the connection between new stellar mass enhancement and instantaneous SFR enhancement is not trivial

$\frac{M_{\text {new stars }}(t)}{M_{\text {new stars, iso }}(t)} \equiv \frac{\int_{0}^{t} \operatorname{SFR}\left(t^{\prime}\right) \mathrm{d} t^{\prime}}{\int_{0}^{t} \operatorname{SFR}_{\text {iso }}\left(t^{\prime}\right) \mathrm{d} t^{\prime}} \neq \int_{0}^{t} \frac{\operatorname{SFR}\left(t^{\prime}\right)}{\operatorname{SFR}_{\text {iso }}\left(t^{\prime}\right)} \mathrm{d} t^{\prime}$.

Changes in nuclear SFR are the result of the combination of changes in SFE (dark pink) and available cold-dense gas fuel (dark blue). The centre experiences strong enhancement in both cold-dense and cool (dark green) gas. SFE in this region is generally enhanced, especially at early times, but reaches sub-unity levels at later times. The outskirts exhibit a deficit in new stellar mass (light brown), caused by suppressed SFR (light purple) during the first $\sim 1.2$ Gyr. SFR recovers eventually, but this is not enough for the mass in new stars to catch up with the isolated galaxy. Generally, this SFR deficit is caused by suppression in both SFE (light pink) and cold-dense gas mass (light blue). The mass content in both cold-dense gas and cool gas (light green) is suppressed for most of the galaxy-pair period, with only a mild recovery at late times.

Unlike the secondary galaxy, the primary (right-hand panels) exhibits new stellar mass and SFR enhancement in both the centre and the outskirts. This enhancement is weak in the outskirts, and weaker in the centre. Even though the centre of this galaxy experiences stronger levels of mass enhancement in cold-dense gas (and cool gas), compared to the centre of the secondary galaxy - especially 

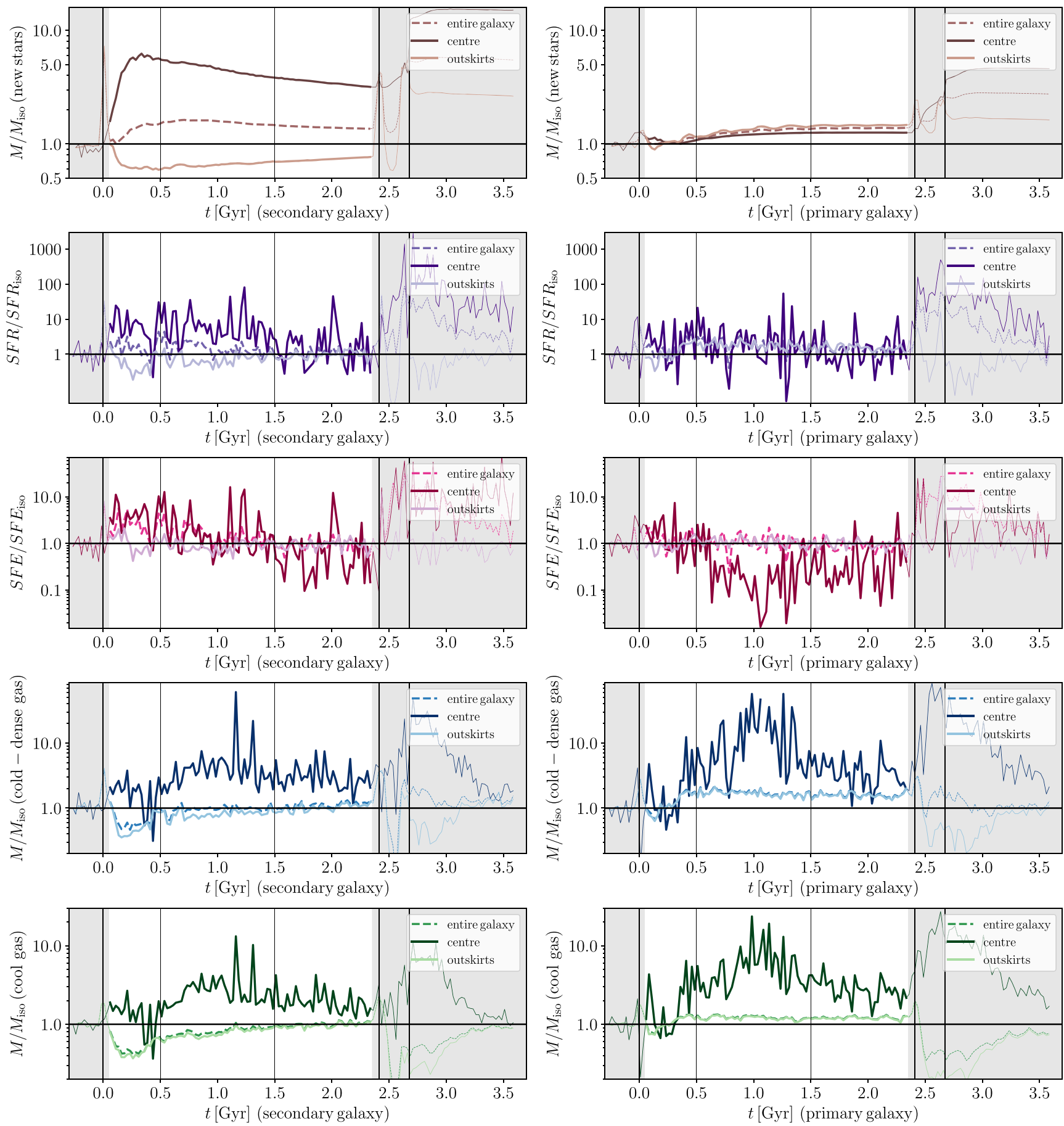

Figure 3. Time evolution of the fiducial run. Left (right-hand) panels: secondary (primary) galaxy. Top-to-bottom panels: new stellar mass (brown), SFR (purple), SFE (pink), cold-dense gas mass (blue), and cool gas mass (green) enhancement. The vertical scales are different for each row. The dark solid lines represent the centre $(0-1 \mathrm{kpc})$, the light solid line represents the outskirts $(1-10 \mathrm{kpc})$, and the medium dashed lines represent the entire galaxy $(0-10 \mathrm{kpc})$. Time is shifted to zero at first pericentric passage. The thick vertical lines from left to right indicate first and second pericentric passages, plus coalescence. The grey boxes and thin portions of the coloured curves represent times outside the galaxy-pair period. The thin vertical lines split the galaxy-pair period into the early $(0-0.5 \mathrm{Gyr})$, intermediate $(0.5-1 \mathrm{Gyr})$, and late periods ( $>1 \mathrm{Gyr})$. The horizontal line indicates unity.

at intermediate times - SFE is strongly suppressed in that region. Section 3.6 explores the connection between SFR, SFE, and colddense gas mass in more detail. In the outskirts, enhanced SFR is driven by an elevated presence of cold-dense gas, while SFE remains close to unity. In other words, both galaxies experience significant increases in cool/cold-dense gas, but SFR does not always increase accordingly.

Fig. 4 tells a similar story for the entire merger suite. The coloured bands are the result of taking the average of quantities described by the coloured lines in Fig. 3 over our 24 mergers 

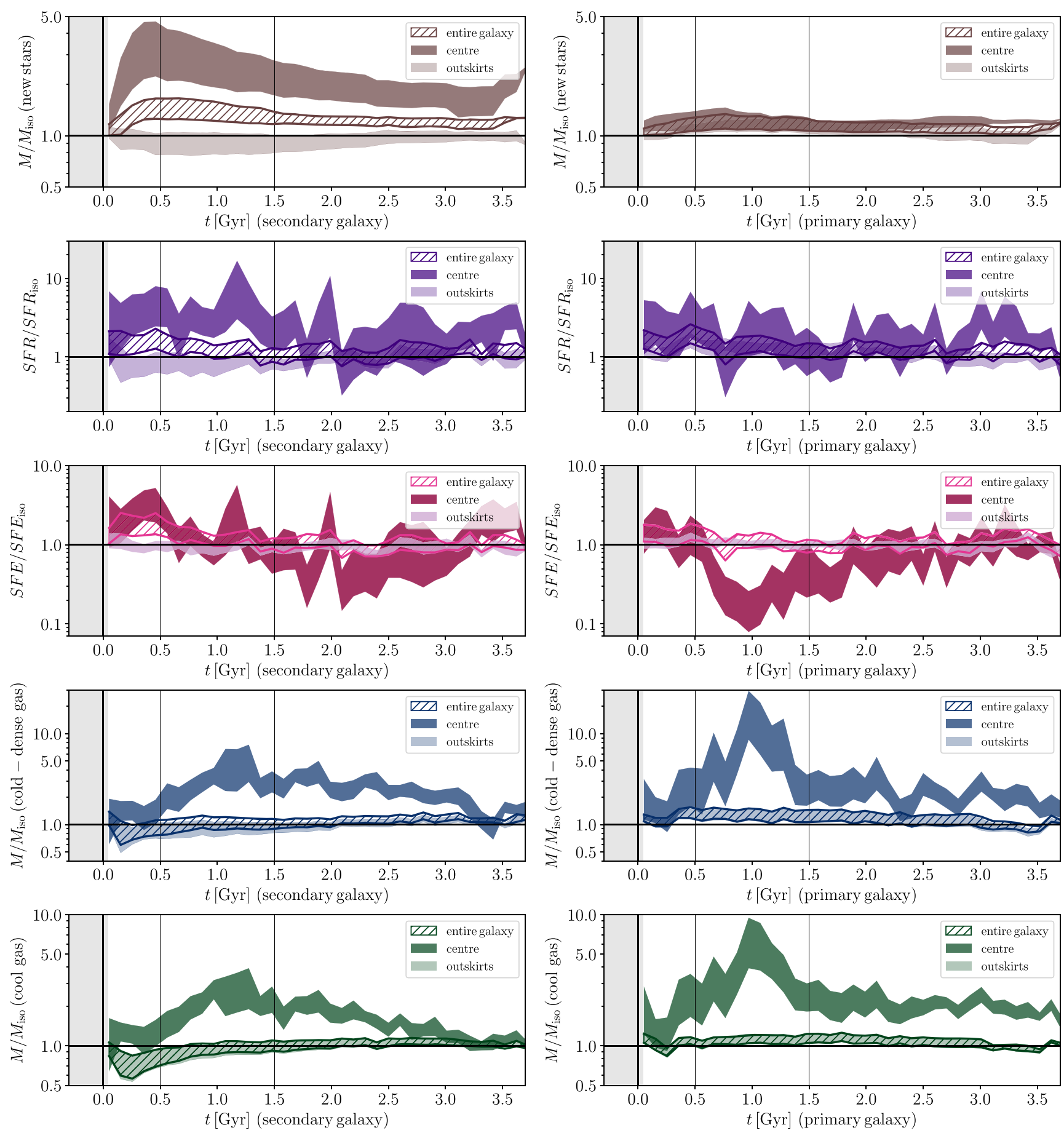

Figure 4. Time evolution for the entire merger suite. Left (right-hand) panels: secondary (primary) galaxy. Top-to-bottom panels: new stars (brown), SFR (purple), SFE (pink), cold-dense gas (blue), and cool gas (green). The vertical scales are different for each row. The dark coloured solid bands represent the centre $(0-1 \mathrm{kpc})$, the light solid bands represent the outskirts $(1-10 \mathrm{kpc})$, and the hatched bands represent the entire galaxy (0-10 kpc). Band thickness corresponds to one standard deviation. Time is shifted to zero at first pericentric passage (thick vertical line). The grey box represents times outside the galaxy-pair period, defined to be between first and second pericentric passage, with separation greater than $20 \mathrm{kpc}$. We do not include lines corresponding to second pericentric passage and coalescence, or the grey box indicating times after the galaxy-pair period, because these vary from merger to merger in the suite. The thin vertical lines split the galaxy-pair period into the early $(0-0.5 \mathrm{Gyr})$, intermediate $(0.5-1 \mathrm{Gyr})$, and late periods $(>1 \mathrm{Gyr})$. The horizontal line indicates unity.

within their respective galaxy-pair periods. Band thickness represents one standard deviation. Note that these panels do not include lines corresponding to second pericentric passage and coalescence. This is because the specific timing of these events varies from merger to merger in the suite. In other words, the late-time regime in this figure is likely to be dominated by contributions from long-lived interactions. See fig. 4 of Moreno et al. (2019) for the diversity in duration and separation extend experienced by the mergers in our suite. In comparing Figs 3 and 4, one notable difference is that, although the fiducial run experiences suppression of star formation 

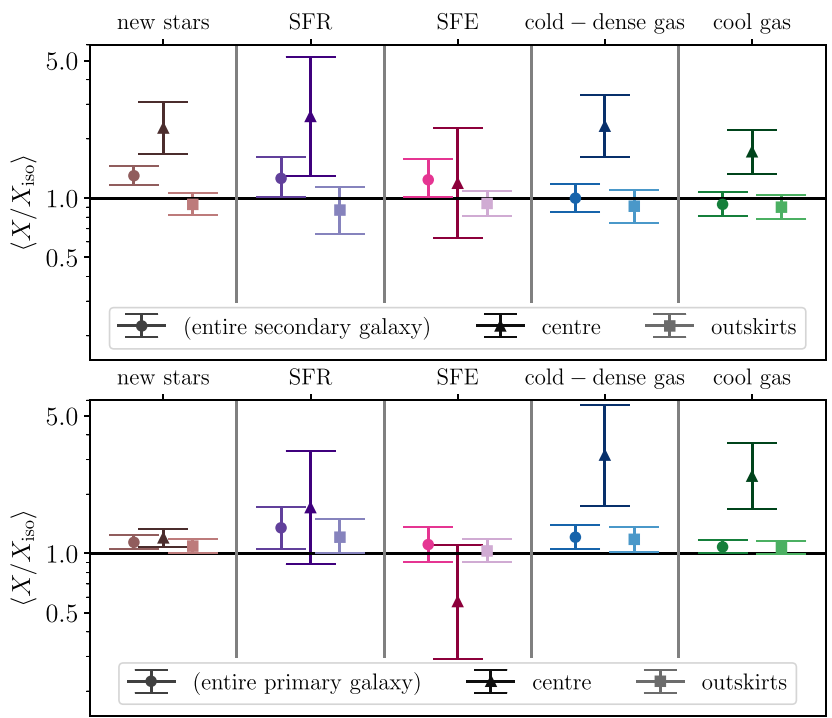

Figure 5. Average sample-wide enhancements across time (within the galaxy-pair period), radial distance (within indicated region), and runs in the merger suite. Top (bottom) panel: Secondary (primary) galaxy. Quantity $X$ represents mass, SFR, or SFE. Left to right symbol clusters: new stars mass (brown), SFR (purple), SFE (pink), cold-dense gas mass (blue), and cool gas mass (green). Left to right within each symbol cluster: entire galaxy (0-10 kpc, medium-tone filled circles), centre (0-1 kpc, dark filled triangles), and outskirts (1-10 kpc, light filled squares). Error bars indicate $1 \sigma$ standard deviations. The vertical grey lines separate symbol clusters. The horizontal black line denotes unity.

between 1 and $10 \mathrm{kpc}$, this effect is not statistically significant when we average over a diverse set of interacting orbits.

Our SFRs are bursty, which is common in our FIRE simulations (Orr et al. 2017; Sparre et al. 2017; Emami et al. 2019; Flores Velázquez et al. 2020). This is strikingly different to predictions by older effective equation-of-state (EOS) models with star formation recipes tuned to match either the Kennicutt (1998) law (Cox et al. 2006; Hayward et al. 2014; Moreno et al. 2015) or a narrow range of SFRs in their isolated galaxies (Di Matteo et al. 2007, 2008). In such models, SFR is gentle (non-bursty) and, after first passage, global SFR is initially enhanced, and becomes heavily suppressed at late times. We do not witness this behaviour in our simulations: at most, global SFR enhancement declines to unity at late times. Making more detailed comparisons is beyond the scope of this paper. We point the interested reader to Hopkins et al. (2013a), who conduct such a comparison between EOS models and a (pre-FIRE) physics model similar to ours with resolved star formation and feedback.

\subsection{Average sample-wide enhancements}

Before studying interaction-induced changes in the radial structure of galaxies, this section reports sample-wide averages. Fig. 5 displays average enhancements across our merger suite. We calculate these values by marginalizing time in the quantities displayed in Fig. 4. The top panel shows results for the secondary galaxy, and the bottom panel for the primary galaxy. Medium-coloured circles, dark triangles, and light squares represent the entire galaxy, the centre, and the outskirts, respectively. Error bars indicate $1 \sigma$ standard deviations. Table 2 presents these values in percentage format.

During the interaction, the secondary galaxy (Fig. 5, top panel) contains, on average, $\sim 30$ per cent more mass in new stars relative to its isolated counterpart. When we focus on the central region,
Table 2. Average sample-wide enhancements. Top (bottom): Secondary (primary) galaxy. Top-to-bottom: new stellar mass, SFR, SFE, cold-dense gas mass, and cool gas mass. Left to right: entire galaxy $(0-10 \mathrm{kpc})$, centre $(0-1 \mathrm{kpc})$, and outskirts $(1-10 \mathrm{kpc})$. Errors refer to $1 \sigma$ standard deviations. Fig. 5 displays these data.

\begin{tabular}{lccc}
\hline Quantity & $\begin{array}{c}\text { Entire galaxy } \\
(0-10 \mathrm{kpc})\end{array}$ & $\begin{array}{c}\text { Centre } \\
(0-1 \mathrm{kpc})\end{array}$ & $\begin{array}{c}\text { Outskirts } \\
(1-10 \mathrm{kpc})\end{array}$ \\
\hline Secondary galaxy & & & \\
New stars & $30_{-14}^{+16}$ per cent & $127_{-50}^{+82}$ per cent & $-7_{-11}^{+13}$ per cent \\
SFR & $26_{-25}^{+36}$ per cent & $160_{-131}^{+263}$ per cent & $-13_{-21}^{+27}$ per cent \\
SFE & $24_{-23}^{+34}$ per cent & $19_{-56}^{+107}$ per cent & $-6_{-13}^{+15}$ per cent \\
Cold-dense gas & $0_{-15}^{+18}$ per cent & $132_{-70}^{+101}$ per cent & $-9_{-16}^{+19}$ per cent \\
Cool gas & $-7_{-12}^{+24}$ per cent & $72_{-39}^{+50}$ per cent & $-10_{-12}^{+14}$ per cent \\
Primary galaxy & & & \\
New stars & $14_{-9}^{+10}$ per cent & $20_{-12}^{+13}$ per cent & $9_{-8}^{+10}$ per cent \\
SFR & $35_{-29}^{+38}$ per cent & $71_{-83}^{+161}$ per cent & $21_{-20}^{+28}$ per cent \\
SFE & $11_{-21}^{+26}$ per cent & $-43_{-28}^{+54}$ per cent & $3_{-13}^{+10}$ per cent \\
Cold-dense gas & $21_{-15}^{+19}$ per cent & $216_{-141}^{+255}$ per cent & $18_{-16}^{+15}$ per cent \\
Cool gas & $8_{-8}^{+9}$ per cent & $147_{-78}^{+115}$ per cent & $7_{-8}^{+9}$ per cent \\
\hline
\end{tabular}

this level of enhancement increases to $\sim 130$ per cent. Some orbital configurations (e.g. our fiducial run) exhibit suppression in the outskirts (defined here as the region spanning radii of $1-10 \mathrm{kpc}$ ) - but on average, this effect is not statistically significant. Instantaneous SFR is mildly enhanced globally ( $\sim 25$ per cent), and strongly enhanced in the centre ( $\sim 160$ per cent). SFR in the outskirts is, on average, statistically consistent with unity. See equation (2) for an explanation of why average enhancements in SFR and new stellar mass are not necessarily identical. SFE is marginally enhanced across the entire galaxy ( $\sim 25$ per cent), but consistent with unity when the centre and outskirts are considered separately. For the cold-dense and cool gas mass budgets, there is also no statistically significant deviation from unity within the outskirts or the entire galaxy. On the other hand, the centre experiences intense levels of mass enhancement: $\sim 130$ per cent in cold-dense gas and $\sim 70$ per cent in cool gas.

Mass in new stars in the primary galaxy (Fig. 5, bottom panel) is enhanced everywhere, not just in the centre. Specifically, the outskirts experience an enhancement of $\sim 10$ per cent. The central and global enhancements are weaker than in the secondary: only $\sim 20$ per cent and $\sim 15$ per cent, respectively. SFR is marginally enhanced in the outskirts ( $\sim 20$ per cent) and globally, and is statistically consistent with unity in the centre. SFE is consistent with unity (within uncertainty) globally, and for both the centre and the outskirts. As in the secondary, the centre exhibits boosts of cold-dense and cool gas mass: $\sim 200$ per cent and $\sim 150$ per cent, respectively. Unlike the secondary, the elevation of cold-dense gas mass in the outskirts is statistically significant: $\sim 20$ per cent. The same is not true for the cool gas phase.

This analysis adds a new layer of detail to the work by Moreno et al. (2019), who report values for the entire system (i.e. the two galaxies combined). These authors report that interacting pairs experience a boost of $\sim 20$ per cent in cold-dense gas and no statistically significant change in cool gas content. Here, we report mass enhancement in both of these gas phases in the centres of both galaxies, and no statistically significant change in the outskirts - except for the cold-dense gas budget in the primary galaxy, which explains the aforementioned trends for the entire system. We warn the reader to interpret average sample-wide results from large sets with care. It is tempting to draw definitive conclusions from simple averages, which often conceal 
subtle but important details. For example, the statement 'new stellar mass suppression in the outskirts is not statistically significant' should not be interpreted as 'new stellar mass suppression never occurs' - it definitely occurs! We certainly do witness this effect in our fiducial run and in many other merging configurations, but just not in the majority of our runs. Furthermore, this result is also sensitive to our rather generous definition of 'outskirts' (i.e. from 1 to $10 \mathrm{kpc})$. Fig. 2 demonstrates that mass suppression of new stars is indeed statistically significant between $\sim 5$ and $8 \mathrm{kpc}$ in the secondary galaxy, and beyond $\sim 8 \mathrm{kpc}$ in the primary - across the entire merger suite! The rest of this paper is devoted to unveiling such details, by exploring how interaction-induced effects depend on properties such as radial location within the galaxy (in finer spatial detail, beyond the centre versus outskirts dichotomy), the time after the first encounter, the geometry of the encounter, and the level of global SFR enhancement.

Observationally, it is now established that galaxy pairs in the local Universe exhibit moderate SFR enhancements, accompanied with strong central enhancements - e.g. Ellison et al. (2013) and Patton et al. (2013), who use the Sloan Digital Sky Survey (SDSS; Abazajian et al. 2009). Using the CO Legacy Database for the GalexArecibo-SDSS (COLD GASS) Survey, Saintonge et al. (2012) report that mergers and morphologically disturbed galaxies tend to have shorter depletion time-scales (equivalent to higher SFEs) than the general population, in line with our predictions (at least for the secondary galaxy). Using IRAM 30-m CO(1-0) observations of SDSS paired-galaxies and controls from the extended COLD GASS (xCOLDGASS) Survey (Saintonge et al. 2017), Violino et al. (2018) find that interactions elevate SFEs (see also Combes et al. 1994). In contrast, Pan et al. (2018) find no interaction-induced deviations in SFE. Neither of these two works compare secondary versus primary galaxies (recall that we predict enhanced global SFE for the former, but not for the latter). Both papers measure interaction-induced $\mathrm{H}_{2}$ mass enhancements in their galaxy pairs, in line with our simulations (at least for the primary galaxy). See also Lisenfeld et al. (2019), who find enhancement in $\mathrm{H}_{2}$ content, but not in SFE. Regarding H I content (equivalent to our cool gas component), observations by Knapen \& James (2009) and Díaz-García \& Knapen (2020) do not find enhancements in atomic gas mass in their galaxy pairs, in line with our global values. We mention, however, that Ellison, Catinella \& Cortese (2018b) report H I enhancement in their postmerger sample.

Cosmological simulations also address interaction-induced star formation. Using Illustris (Vogelsberger et al. 2013), EAGLE (the Evolution and Assembly of GaLaxies and their Environments project; Schaye et al. 2015), and IllustrisTNG (Pillepich et al. 2018) - Patton et al. (2020) demonstrate that SFR is enhanced in galaxies with relatively close companions relative to carefully matched noninteracting controls (mimicking the observational methodology of Patton et al. 2013, 2016), and that this is a generic feature across simulations with very distinct physics assumptions. This result confirms earlier findings with smaller simulation volumes (Perez et al. 2006) - but see Tonnesen \& Cen (2012), who do not identify enhanced specific SFR (sSFR) in their cosmologically selected galaxy pairs with separations below the Roche limit. Using SIMBA (Davé et al. 2019), Rodríguez Montero et al. (2019) investigate what drives elevated SFR in mergers. They find that for low-mass galaxies (stellar mass below $10^{10.5} \mathrm{M}_{\odot}$ ), increases in SFR are attributed to increases in $\mathrm{H}_{2}$ content, not $\mathrm{SFE}$ - and that this trend reverses for more massive galaxies. Surprisingly, we find that for the secondary galaxy, global SFR enhancement is driven by enhanced SFE (Fig. 5, top panel, medium purple and pink circles) - while, for the primary, it is driven by enhanced cold-dense gas content (Fig. 5, bottom panel, medium purple and green circles). Unfortunately, these authors identify mergers by selecting systems with sudden jumps in stellar mass (above that expected from in situ star formation), which may include both galaxy-pairs and mergers past the coalescing period. Interestingly, Sparre \& Springel (2016) use zoom-in simulations from Illustris to highlight the importance of making this distinction: during the galaxy-pair period, SFR enhancement is driven by increases in $\mathrm{H}_{2}$ content - but after coalescence, it is driven by increases in SFE.

\subsection{Radial structure and evolution}

The previous section studies time evolution in three radial regions: the centre $(0-1 \mathrm{kpc})$, the outskirts $(1-10 \mathrm{kpc})$, and the entire galaxy $(0-10 \mathrm{kpc})$. In this section, we dissect radial structure in finer detail, at the expense of having to use cruder time bins: the early $(0<t<$ $0.5 \mathrm{Gyr})$, intermediate $(0.5<t<1.5 \mathrm{Gyr})$, and late $(t>1.5 \mathrm{Gyr})$ period of interaction. See Section 2 for a justification, and the thin vertical lines in Figs 3 and 4 for reference. We focus on the fiducial run only, and explore other merging configurations in Section 3.4. We present three baryonic components: new stars, cold-dense gas, and cool gas - and defer an analysis of the spatial extent of SFR and SFE to Section 3.5. Fig. 6 displays these average profile ratios. The hatched bands represent the entire galaxy-pair periods, while the coloured bands (from light-to-dark) represent averages constraint to times in the early, intermediate, and late periods.

For the secondary galaxy (left-hand panels), enhancement in new stellar mass is centrally peaked (within $\sim 2 \mathrm{kpc}$ ), and suppressed at larger galactocentric radii. This behaviour is particularly accentuated during the early period, and becomes weaker at later times. The cold-dense and cool components behave similarly, except that enhancement does not peak at the centre, and suppression starts at different radii: $\sim 4.5 \mathrm{kpc}$ and $\sim 5.5 \mathrm{kpc}$, respectively. Recall that suppression at the centre does not necessarily imply the presence of a 'hole' in the gas distribution, but rather it is commonly attributed to a diminishment in mass relative to the isolated control galaxy (e.g. Fig. 1, fourth-row/third-column panel). As a function of time, the peak shifts outwards (from 0 to $\sim 0.8 \mathrm{kpc}$ ) and suppression at larger radii becomes weaker. At early times, both new stars and cold-dense gas also exhibit an uptick at the very largest radii. By inspecting a video from which the images in Fig. 1 were drawn, we find that this effect is explained by material that was originally launched into tidal tails and a bridge after the first encounter (D'Onghia et al. 2010; Blumenthal \& Barnes 2018), becomes compressed there, and is now settling on to the outer portions of the disc. This phenomenon is a modern version of the sequences of events described by the original Barnes \& Hernquist (1996) paper - but here we employ a model that resolves the structure of the ISM. In a future paper, we investigate how cold-dense gas and new stars are formed in tidal tails and bridges, and later migrate back on to the discs.

The radial structure of mass enhancement in new stars in the primary galaxy (Fig. 6, right-hand panels) is strikingly different. On average, two peaks are formed: one spanning $\sim 0-0.7 \mathrm{kpc}$, and the other spanning $\sim 0.8-7 \mathrm{kpc}$. Initially, this distribution is weak and flat, but it gets stronger around these two radii at later times as new stars are born. The cold-dense and cool gas components exhibit strong enhancement between $\sim 0$ and $2 \mathrm{kpc}$, accompanied by a milder plateau that extends out to $\sim 7 \mathrm{kpc}$, with suppression beyond that radius. Initially, the plateau is weak and extends all the way to the centre. At later times, the above secondary concentration at small radii becomes stronger and its peak shifts to slightly larger radii. This extended reservoir of cold-dense (and cool) gas explains the 

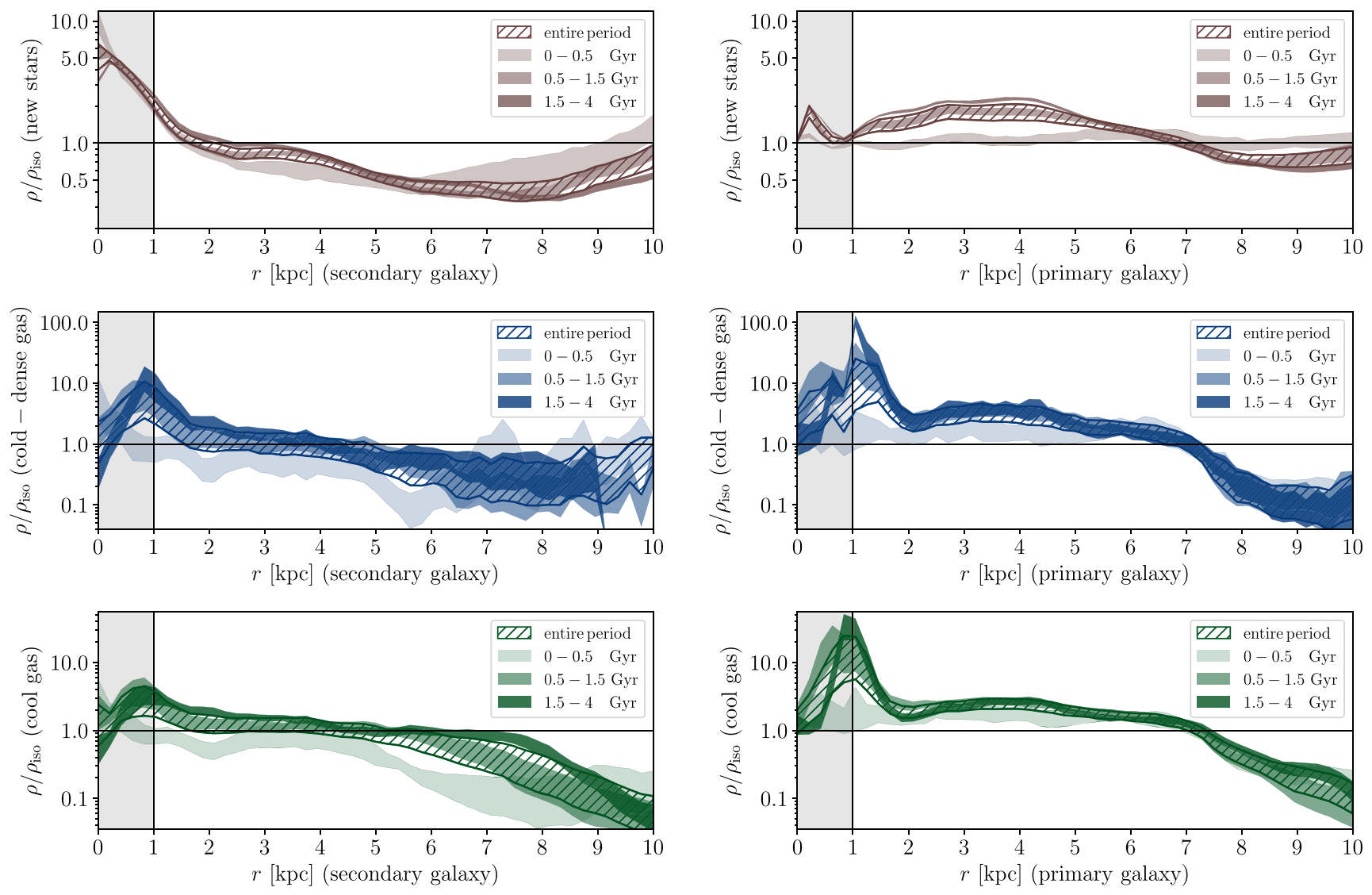

Figure 6. Average profile ratios for the fiducial run. Left (right-hand) panels: secondary (primary) galaxy. Top-to-bottom panels: new stars (brown), cold-dense gas (blue), and cool gas (green). The hatched band denotes entire galaxy-pair period, defined to be between first and second pericentric passage, with separation greater than $20 \mathrm{kpc}$. The light, medium, and dark coloured bands represent early $(0-0.5 \mathrm{Gyr})$, intermediate $(0.5-1.5 \mathrm{Gyr})$, and late ( $>1.5 \mathrm{Gyr})$ times (within the galaxy-pair period). Band thickness refers to one standard deviation. The vertical black line and grey box highlight central region $(r<1 \mathrm{kpc})$. The horizontal line represents unity.

build-up of the corresponding plateau in new stars (Fig. 6, top-righthand panel). In contrast to the secondary galaxy, the primary has a stronger increase in cold-dense (and cool) gas mass at small radii. This does result in a increase in new stars in that region, but this boost is weaker than in the centre secondary galaxy. The presence of new stars is the cumulative effect of SFR, which in turn is governed by the amount of fuel available and SFE. Recall that SFE is suppressed in the centre, and remains close to unity in the outskirts (Fig. 3, third-row/second-column panel, dark versus light pink). We address the connection between SFE, available cold-dense gas fuel, and SFR in detail in Sections 3.5 and 3.6.

There are very few statistical studies on the spatial extent of interaction-induced star formation and molecular-gas fuelling using observations in the local Universe. Barrera-Ballesteros et al. (2015a) use a sample of over 100 merging galaxies drawn from the CALIFA survey (Sánchez et al. 2012), and report sSFR enhancement in the central region, and moderate-to-null suppression in the outskirts, in line with our findings. These authors do not report results in terms of primary versus secondary galaxy. Similarly, using a MaNGA data set containing over 200 merging galaxies, Pan et al. (2019) split their sample into four evolutionary stages, two of which are directly relevant to our work: 'Stage 2' (corresponding to our early period) and 'Stage 3' (corresponding to our intermediate and late periods). Their observations suggest tantalizing similarity with our results: steep centrally concentrated star formation, with decaying excess profiles at large galactocentric radii; and with a decrease in central steepness at later times. Direct comparison with their work is difficult for two reasons: (1) they only probe out to 1.5 half-light radii, and (2) they do not split their sample into primary and secondary galaxies. See also Thorp et al. (2019), who perform a similar analysis for the post-merger period, and Ellison et al. (2018a) for more general (nonmerger specific) results. Interestingly, in the stellar-mass regime we cover here, Spindler et al. (2018) find that satellites exhibit enhanced star formation in the centre and suppression in the outskirts relative to centrals. If this result remains true for systems where the satellite is (1) the most massive satellite within the host dark matter halo, and (2) it is interacting with the central - these observations would be consistent with our predictions (Fig. 6, top-left versus top-right panel).

To investigate the spatial extent of the cold molecular gas component (our 'cold-dense' gas phase), Yamashita et al. (2017) use COobservations with the Nobeyama Radio Observatory on a sample of 58 interacting galaxies drawn from the Great Observatories All-sky LIRGs (Luminous Infrared Galaxies) Survey (GOALS; Armus et al. 2009). These authors find that, on average, the CO radii of galaxies in widely separated pairs is larger than their isolated counterparts, and become smaller as they approach second pericentre. This is contrary to our findings (at least for the secondary galaxy, Fig. 6, bottom-left panel, light-to-dark bands). However, these authors also normalize their CO-sizes relative to the size of the stellar component, which may also experience changes in physical size during the interaction 
(Moreno et al., in preparation). Direct comparison with our work is also challenging because they focus on extreme systems (LIRGS), while our suite is designed to model more common galaxy interactions found in the SDSS. To empirically corroborate this behaviour, $\mathrm{CO}$-observations of a larger set of interacting galaxies (beyond LIRGs) with more finely sampled evolutionary stages is required.

\subsection{Dependence on orbital merging geometry}

So far we have only reported average profile ratios for the fiducial run. Generalizing these results to all mergers in the suite is challenging because one needs to disentangle variations caused by time evolution within an individual merger and differences amongst various merger configurations. Simply averaging across the entire suite (e.g. as in Fig. 2) might mask subtle, but important, details. It is not practical to repeat the analysis presented in Fig. 6 for all 24 mergers in the suite. Rather, we visually inspect these single-run average profile ratios for the three baryonic components displayed in Fig. 6 (new stars, cool gas, and cold-dense gas), and group them together if they share common features. We find that we can split the 24 configurations in our suite into three main categories, or subsuites:

(i) The typical subsuite includes every near-prograde configuration (8 mergers), plus those near-polar configurations with first pericentric passages at intermediate $(\sim 16 \mathrm{kpc})$ and large $(\sim 27 \mathrm{kpc})$ separations (5 mergers), and those near-retrograde with small separation ( $\sim 7 \mathrm{kpc}$ ) at first pericentric passage ( 3 mergers). This amounts to $16 / 24$ mergers, or 66.7 per cent of the entire suite. The fiducial run belongs to this subsuite.

(ii) The vigorous subsuite includes near-polar configurations with first pericentric passage at $\sim 7 \mathrm{kpc}$. This amounts to only $3 / 24$ mergers, or 12.5 per cent of the suite.

(iii) The gentle subsuite contains those near-retrograde configurations with intermediate $(\sim 16 \mathrm{kpc})$ and large $(\sim 27 \mathrm{kpc})$ separations at first pericentric passage. This corresponds to 5/24 mergers, or 20.1 per cent of the suite.

We adopt the terms 'typical', 'vigorous', and 'gentle' to informally describe how encounters in these subsuites alter the average profile ratios (for the three baryonic components) with respect to unity during the galaxy-pair period - i.e. this naming scheme might not be appropriate to describe the impact of these orbital geometries on radial structure after coalescence. We emphasize that we choose to group our mergers in these three subsuites as an alternative to the cumbersome presentation of 24 individual configurations. There is no a priori rigorous physical reason why we should group our orbits in this particular fashion. At the time of writing, we are not aware of any published work where merger simulations are segregated in this manner. Commonly, authors group orbits in terms of spin-orbit orientation only (e.g. Di Matteo et al. 2007; Moreno et al. 2015). Here, we note that the combination of orientation, and whether or not the two discs intersect, governs how radial structure evolves after first pericentric passage. Investigating dynamical processes in this context is the subject of future work.

Fig. 7 displays configurations in the typical, vigorous, and gentle subsuites using forward-slash, vertical, and back-slash hatched bands, respectively. We calculate these average profile ratios by taking the average of individual profile ratios across every member configuration of each subsuite, and across their respective galaxy-pair periods. Note that the results corresponding to the typical subsuite resemble those corresponding to the fiducial run (hatched bands here versus hatched bands in Fig. 6). Below we describe features appearing in the vigorous and gentle subsuites in detail, and compare them to those in the (larger) typical subsuite (which contains the fiducial case - discussed thoroughly already).

The secondary galaxy (left-hand panels) displays the most dramatic effects in the vigorous subsuite. New stellar mass is suppressed severely between $\sim 0.5$ and $8.5 \mathrm{kpc}$. The cold-dense and cool gas mass budgets are also strongly suppressed between $\sim 1-5$ and $\sim 1-$ $9 \mathrm{kpc}$, respectively. Interacting galaxies in the gentle subsuite, on the other hand, experience the mildest effects. Galaxies in this category experience weak mass enhancement in new stars within $\sim 1.5 \mathrm{kpc}$ and beyond $\sim 6 \mathrm{kpc}$. The cold-dense and cool gas mass budgets are elevated within the central $\sim 0.5 \mathrm{kpc}$, and beyond $\sim 8$ and $\sim 7 \mathrm{kpc}$, respectively. The primary galaxy (right-hand panels) in the vigorous subsuite acts as a more-intense version of the typical subsuite, with slightly larger central baryonic concentrations, and spatially extended plateaus. Overall, average profile ratios belonging to the gentle subsuite tend to be flat and close to unity, with dips between $\sim 3$ and $6 \mathrm{kpc}$ for the new stellar component, and near $\sim 1.5 \mathrm{kpc}$ for the cold-dense and cool gas components.

Very little numerical work exists on how the spatial distribution of new stars and cold-dense/cool gas depends on orbital merging geometry. Di Matteo et al. (2008) present surface density maps of SF gas for a handful of time frames for two mergers, one with prograde and one with retrograde spin-orbit orientation. Their prograde merger exhibits large concentrations of gas and SF regions in the centres, with secondary contributions from tidal tails, the bridge, and ring structures (see also Moreno et al. 2015, who report the existence of SF rings in their EOS simulations). Their retrograde merger only exhibits strong gas concentrations and intense star formation in the centres. These authors do not present a quantitative analysis like ours, making a direct comparison with their work unfeasable. Moreno et al. (2015) split their sample into three subsuites of identical size: the near-prograde, near-polar, and near-retrograde orientations. We use exactly the same spin-orbit orientations as in that paper, but with a new model (FIRE-2). In that paper, the authors find that star formation in the secondary galaxy is enhanced in the centre, and suppressed in the outskirts. This effect is particularly strong for near-prograde and near-polar orbits, and weak for near-retrograde configurations. We find similar trends when we compare the typical subsuite (containing all the near-prograde orbits) against the gentle subsuite (containing most of the near-retrograde mergers): enhancement in the centre and suppression at large galactocentric radii is more pronounced in the former category than in the latter one (if at all). Unlike those older simulations, we generally find that our levels of enhancement and suppression are weaker, and suppression appears at larger radii: beyond $\sim 6 \mathrm{kpc}$ in this work, versus $\sim 1 \mathrm{kpc}$ in Moreno et al. (2015). In that older work, the primary galaxy exhibits strongly boosted star formation in the centre for the near-prograde interactions, and weak enhancement out to larger radii (out to $\sim 3 \mathrm{kpc}$ for near-polar orbits, and everywhere for near-retrograde orbits). The primary galaxy in our FIRE-2 simulations produces more new stars in the typical subsuite than in the gentle subsuite. The former is weakly enhanced within $\sim 6 \mathrm{kpc}$, while the latter is weakly enhanced within $\sim 1 \mathrm{kpc}$. Overall, our results suggest that incorporating resolved, feedback-regulated, physics - and their effect on the turbulent structure of the ISM serves to mitigate the pronounced centre-versus-outskirts disparity prevalent in older models.

At the time of writing, we do not find any observational work exploring the connection between orbital orientation and the spatial extent of new stars and cold-dense/cool gas. To address connections between orbital orientation and global properties, Mesa et al. (2014) use a sample of $\sim 1500$ visually classified galaxy pairs selected from the SDSS. They use spiral-arm direction to break their sample 

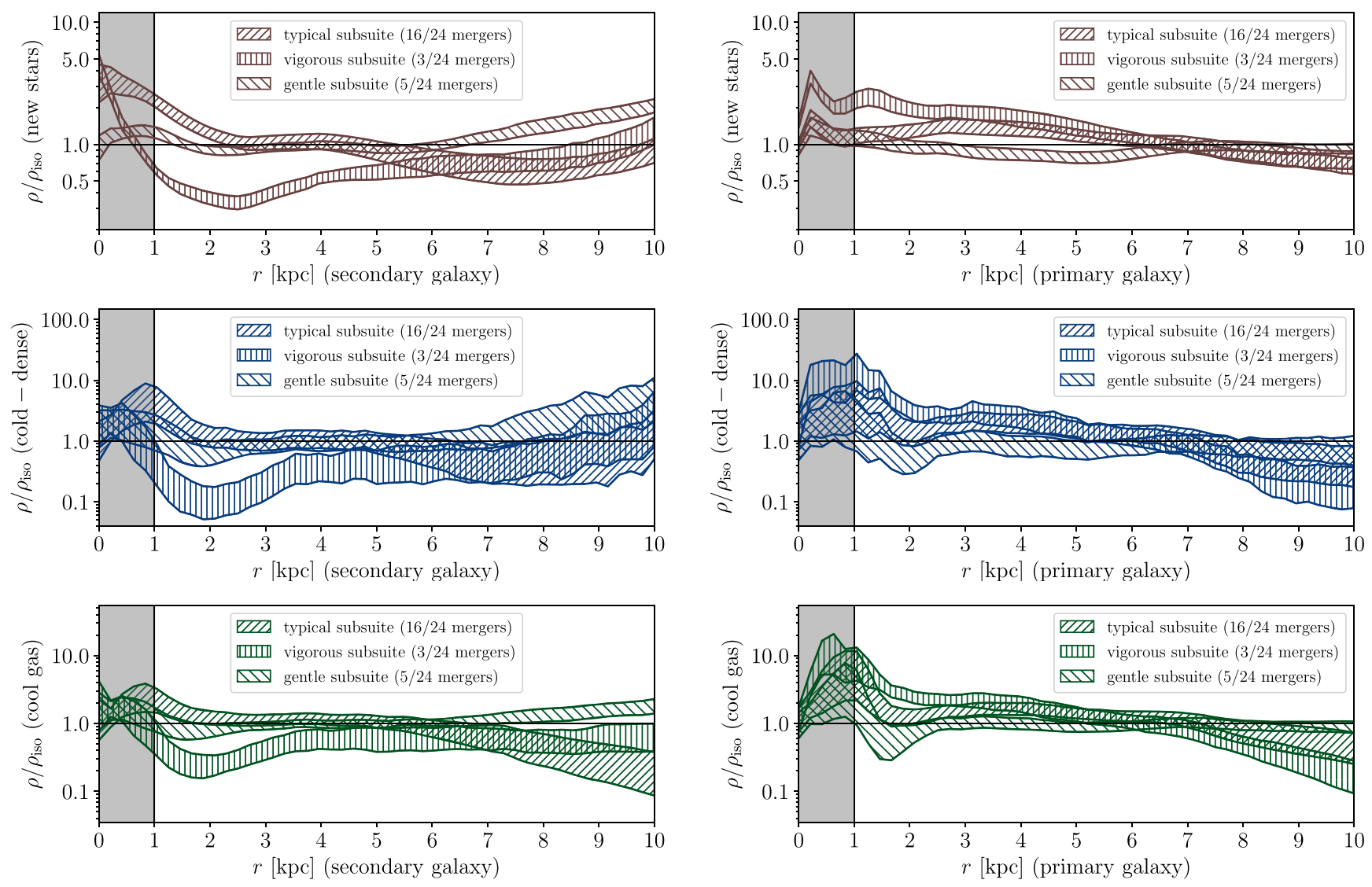

Figure 7. Average profile ratios for three subsets of galaxy-merger configurations (subsuites). Left (right-hand) panels: secondary (primary) galaxy. Top-tobottom panels: new stars (brown), cold-dense gas (blue), and cool gas (green). The forward-slash, vertical, and backward-slash hatched bands represent the typical, vigorous, and gentle subsuites (keys indicate fraction of mergers in each subsuite - see Section 2 for definitions). Band thickness refers to one standard deviation. The vertical black line and grey box highlight the central region $(r<1 \mathrm{kpc})$. The horizontal line represents unity. Galaxy-pair periods only.

into subsamples of co-rotating and counter-rotating pairs. These authors report bluer colours and younger stellar populations in their counter-rotating systems. To compare directly with our work, it would be interesting to follow up these systems with spatially resolved IFU observations, which would allow the measurement of the spatial structure of the SF component as a function of orbital orientation. Comparing the results from such an exercise with our work presents two caveats. (1) The Mesa et al. (2014) SDSS sample only selects galaxies displaying tidal tails. Inspection of videos of our simulations (analysis not included here) show that such selection is biased towards the early period (second-row/first-column image versus other second-row images in Fig. 1). This claim is consistent with findings by Blumenthal et al. (2020), who use a cosmological simulation of galaxy formation (IllustrisTNG; Pillepich et al. 2018) to infer that only $\sim 45$ per cent of interacting pairs display visually identified features. Lastly (2), our results suggest that it is not enough to break our suite by spin-orbit orientation alone (as in Moreno et al. 2015) - but information on whether or not the two disc interpenetrate one another at first pericentric passage is just as pertinent. We explore these details in future work.

\subsection{Global SFR enhancement versus radial structure}

It has been known for almost two decades that SF galaxies follow a tight SFR $-M_{\star}$ relation, known as the SF 'main sequence' (SFMS; Brinchmann et al. 2004; Noeske et al. 2007; Elbaz et al. 2007;
Whitaker et al. 2012; Salim et al. 2014). Following this discovery, it has been suggested that regions above this sequence (i.e. the starburst regime) tend to be populated by merging systems (Jogee et al. 2009; Hung et al. 2013; Willett et al. 2015; Pearson et al. 2019). However, the majority of these systems tend to be late-stage mergers, with galaxy pairs spending more time in regions closer to the main ridgeline of this relation (Puech et al. 2014; Cibinel et al. 2019). Conversely, observations by Silva et al. (2018) show that only 12-20 per cent of close galaxy pairs with separations between 3 and $15 \mathrm{kpc}$ are starbursts, depending on which stellarmass bin is considered. Likewise, using a cosmological simulation (IllustrisTNG; Pillepich et al. 2018), Blumenthal et al. (2020) show that interacting galaxies in the pre-merger phase are more likely to lie well above the SFMS only at or soon after their most recent close pericentric passage. Similarly, Wilkinson et al. (2018) use the original Illustris (Vogelsberger et al. 2013) to show that $\sim 55$ per cent of starburst in the local Universe are activated by tidal interactions with their neighbours.

The emergence of spatially resolved integral-field unit (IFU) surveys has stimulated an interest in how the internal structure of a galaxy is connected to its location relative to the SFR- $M_{\star}$ relation (e.g. Sánchez et al. 2012; Cano-Díaz et al. 2016; González Delgado et al. 2016; Hsieh et al. 2017). This motivates us to analyse the connection between global SFR enhancement and radial structure in interacting galaxies. To continue teasing out the effects caused by the encounter, here we elect to compare against the SFR/SFR iso $=1$ line, not the SFMS. Fig. 8 splits our sample into three types of SFs: 

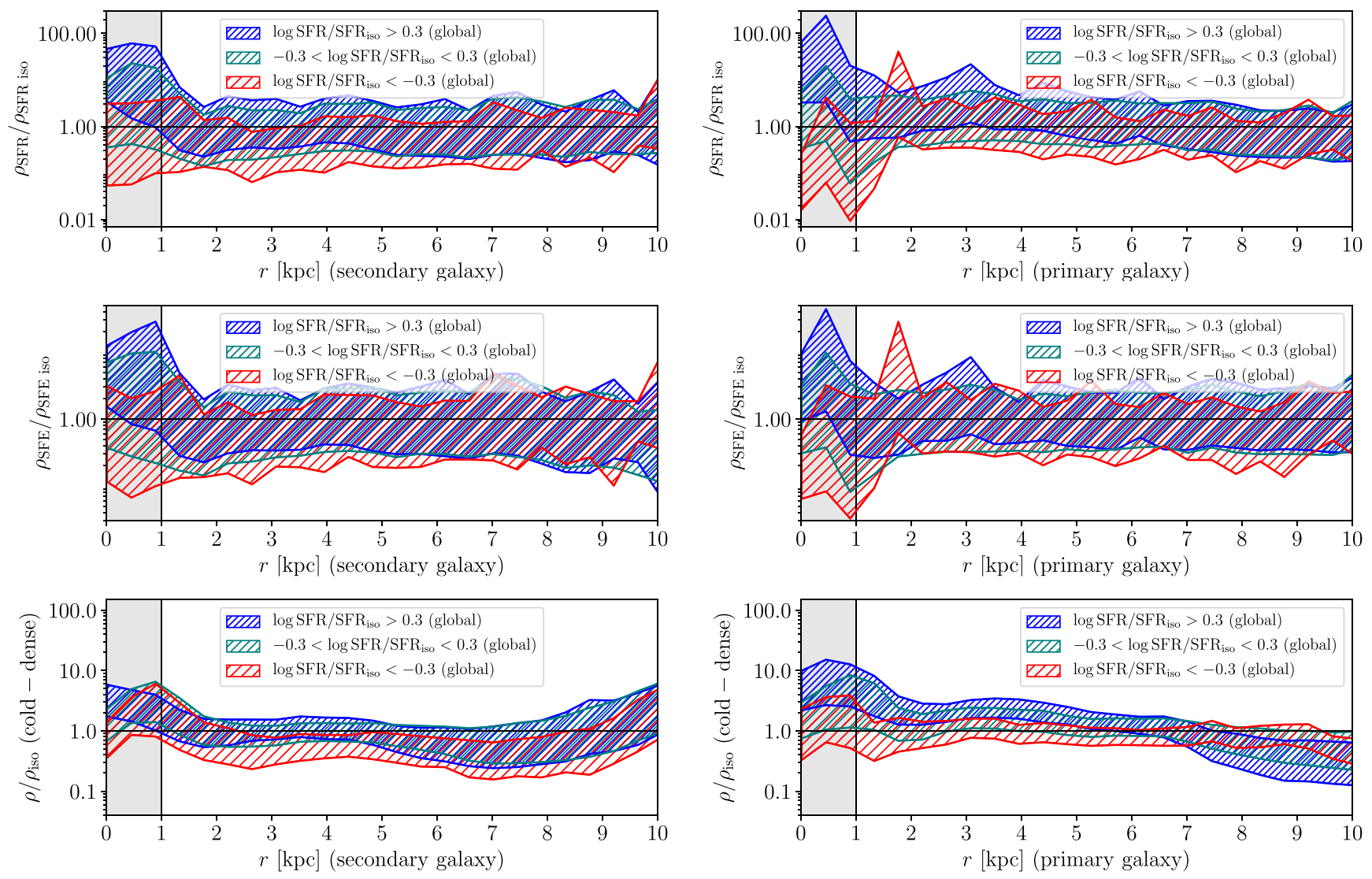

Figure 8. Average profile ratios split into three global SFR enhancement bins. Left (right-hand) panels: secondary (primary) galaxy. Top-to-bottom panels: instantaneous SFR, SFE, and cold-dense gas mass. The densely packed (blue), regular (green), and sparsely packed (red) hatched bands represent the result of averaging over galaxies with global (within $10 \mathrm{kpc}$ ) SFR enhancement greater that +0.5 dex, between -0.5 and +0.5 dex, and below -0.5 dex, respectively. Band thickness refers to one standard deviation. The vertical black line and grey box highlight the central region $(r<1 \mathrm{kpc})$. The horizontal line represents unity. Galaxy-pair periods only. Table 3 lists percentages per SF type and galaxy.

Table 3. Types of SFs: definitions and percentages per galaxy. SFR/SFR iso denotes global SFR enhancement. Fig. 8 shows radial structure per SF type in terms of SFR, SFE, and cold-dense gas mass.

\begin{tabular}{lcrr}
\hline SF type & Definition & Secondary & Primary \\
\hline Enhanced & $\log \mathrm{SFR} / \mathrm{SFR}_{\text {iso }}>+0.3$ & 15.2 per cent & 16.9 per cent \\
Regular & $-0.3<\log \mathrm{SFR} / \mathrm{SFR}_{\text {iso }}<+0.3$ & 82.5 per cent & 81.5 per cent \\
& $\log \mathrm{SFR} / \mathrm{SFR}_{\text {iso }}<-0.3$ & 2.3 per cent & 1.6 per cent \\
Suppressed & & & \\
\hline
\end{tabular}

enhanced SFs (at least 0.3 dex above unity, blue closely hatched), regular SFs (between -0.3 and +0.3 dex from unity, green hatched), and suppressed SFs (at least 0.3 dex below unity, red loosely hatched). We choose 0.3 dex as a compromise between the 0.2 dex half-scatter of the SFMS (Belfiore et al. 2018) and the 0.5-0.6 dex thresholds commonly adopted to identify starbursts (e.g. Wilkinson et al. 2018; Ellison et al. 2020a). We note that our galaxies are predominantly regular SFs at the great majority of times ( $\sim 80$ per cent). See Table 3 for a list of percentages per SF type and galaxy. Note that individual galaxies are not permanent members of any specific SF type - rather, they may 'visit' all three regimes throughout the duration of their interaction (Martínez-Galarza et al. 2016). Also, we emphasize that we quote enhancements relative to their respective isolated galaxies, not to the global SFMS.

For the secondary galaxy (Fig. 8, left-hand panels), the average SFR profile ratios (top panels) for the three SF-type bins are similar at large radii, and diverge towards the centre. The large scatter displayed by the thickness of all three bands (corresponding to one standard deviation) is driven by the bursty nature of star formation in our FIRE-2 physics model (e.g. Orr et al. 2017). Globally enhanced SFs experience elevated SFR within $\sim 0.9 \mathrm{kpc}$. This is caused by the combination of enhanced SFE (middle panels) and elevated colddense gas content (bottom panels) in that region. The regular SFs also experience a central boost in cold-dense gas mass, but not in SFE, causing SFR to be consistent with unity in that region. The globally suppressed SFs, on the other hand, exhibit a flat average SFR profile ratio at most radii, except near $\sim 2.5-3 \mathrm{kpc}$, where it dips below unity. This is driven by a deficit of cold-dense gas at those radii, which prevails over the lack of SFE suppression in that region.

The primary galaxy (Fig. 8, right-hand panels) also experiences a bifurcation of average SFR profile ratios near the centre: globally enhanced SFs bend upwards, while their suppressed counterparts bend downwards with decreasing radius (in the case of the secondary, the globally suppressed SFs bend downward relative to the enhanced SFs, but not relative to unity). The primary experiences a boost in cold-dense gas mass within $\sim 5 \mathrm{kpc}$ for the globally enhanced $\mathrm{SF}$ population. However, SFE is only enhanced within $\sim 0.5 \mathrm{kpc}$, which explains why SFR enhancement occurs only within that smaller region. SFR enhancement near $\sim 3 \mathrm{kpc}$, on the other hand, is explained by excess in cold-dense gas (SFE is consistent with unity there). For the globally suppressed SF sample, the deficit in SFR 
within $\sim 0.3 \mathrm{kpc}$ is explained by suppressed SFE alone - cold-dense gas content is consistent with null enhancement in that region.

The upward versus downward bifurcation discussed above is observed by IFU surveys for galaxies with global SFR above and below the SFMS. Using SAMI, Medling et al. (2018) find that SFR surface density $\left(\Sigma_{\mathrm{SFR}}\right)$ profiles bend downward in the inner regions of galaxies inhabiting the regime located $1-3 \sigma$ below the SFMS. Belfiore et al. (2018) find similar results with MaNGA: the $\Sigma_{\text {SFR }}$ profiles of galaxies in the Green Valley - i.e. those located $1 \sigma(0.39$ dex) below the SFMS, experience a downward turn near the centre (see also Brownson et al. 2020). Using the SIMBA cosmological simulation (Davé et al. 2019), Appleby et al. (2020) explain this trend as the combination of two effects: lower $\mathrm{H}_{2}$-gas mass presence towards smaller radii, and a decrease in SFE in that region. In contrast, our simulations predict a downturn in SFE (primary galaxy only), and no statistically significant change in cold-dense gas content (Fig. 8, middle and bottom panels, loosely hatched red bands). For a more direct comparison with SIMBA, it would be interesting if those authors performed an analysis of paired-versus-isolated galaxies similar to ours.

Using EDGE-CALIFA (Bolatto et al. 2017), Chown et al. (2019) find that galaxies with $\mathrm{H} \alpha$ inner upturns tend to be barred galaxies, mergers, or galaxy in close pairs. However, not all mergers/pairs have such upturns. Also, they find that galaxies with upturns tend to have higher molecular-gas concentrations, but the presence of such fuel reservoirs are not always required for galaxies with upturns. This observational study does not separate galaxies in terms of their location to the global SFMS - or relative to non-interacting controls (in the case of pairs). Ellison et al. (2018a) report both downturns and upturns towards the smaller radii in their MaNGA-selected $\Sigma_{\text {SFR }}$ profiles at low galactocentric radii for galaxies with SFR above and below their resolved (spaxel-by-spaxel) SFR $-M_{\star}$ relation (the 'rSFMS' - see e.g. Ellison et al. 2018a; Hani et al. 2020a). Wang et al. (2019) find similar results with the same survey, but using the median $\Sigma_{\mathrm{SFR}}$ in stellar-mass bins (rather than the rSFMS) as reference. Morselli et al. (2019) report similar bifurcations out to $z$ $=1.2$ using multiwavelength Hubble Space Telescope (HST) data selected from the GOODS+CANDELS campaign. ${ }^{4}$ Using a sample of 12 starbursts selected from ALMaQUEST (Lin et al. 2019), Ellison et al. (2020a,b) find that galaxies well above the global SFMS exhibit upturns in their SFE profiles, in agreement with our results (Fig. 8, middle panels, densely packed blue versus loosely hatched red bands). We also predict these galaxies to have an upturn in colddense gas mass at lower galactocentric radii (Fig. 8, middle panels). Instead, ALMaQUEST starbursts exhibit a mix of $f_{\mathrm{H}_{2}}$ profile shapes, many of which bend downwards, with suppressed $\mathrm{H}_{2}$ mass near the centre.

While encouraging, comparisons between the above observations and our simulations must be interpreted with care. First, we do not compare profiles relative to systems on the SFMS (or the rSFMS), but rather relative to profiles associated with identical galaxies simulated in isolation. Although we do not expect this correction to cause substantial effects (our isolated galaxies remain close to the global SFR $-M_{\star}$ relation), discrepancies may be amplified when our isolated control galaxies momentarily deviate from the SFMS due to secular processes. Secondly, samples drawn from spatially resolved surveys - especially those with follow-up observations using interferometric

${ }^{4}$ GOODS stands for Great Observatories Origins Deep Survey, while CANDELS stands for Cosmic Assembly Near-infrared Deep Extragalactic Legacy Survey. arrays - tend to be small. The above sections demonstrate that the shapes of density profiles depend not only on global SFR, but also on the time of observation (Fig. 6) and the orbital geometry (Fig. 7). Thus, it is possible that recently published observed samples are not necessarily representative. For these reasons, it is critical that the next generation of spatially resolved galaxy surveys (1) expand campaigns similar to that conducted by Pan et al. (2019) - who compare spatially resolved interacting systems against properly matched controls - but with substantially larger and more varied galaxy samples; and (2) that these programmes are coupled with follow-up interferometric observations to infer the role of SFE, molecular gas content, and their spatial structure.

Our results suggest that, in a few ways, the inner regions of our interacting systems in the local Universe resemble high-redshift galaxies experiencing 'compaction': i.e. disc-contraction episodes believed to be driven by cold streams and mergers, producing starbursting 'blue nuggets' as a result (Dekel \& Burkert 2014; Zolotov et al. 2015). Concretely, when we segregate interacting galaxies in terms of their global SFR, we find a bifurcation in SFR, SFE, and available fuel at small galactocentric radii. At high redshift, galaxies in the upper envelope of the SFMS exhibit high gas fractions, high SFEs, and cuspy gas profiles - while those in the lower envelope are endowed with low gas-fractions, low SFEs, and gas-depleted cores (Tacchella et al. 2016a,b). Investigating the importance of the relative contribution of galaxy-galaxy interactions to compaction and quenching in the local Universe (Woo \& Ellison 2019) is the subject of future work.

\subsection{What drives star formation in the central kiloparsec?}

The previous section demonstrates that the strongest interactioninduced modifications to radial SFR structure occur at small galactocentric radii (Fig. 8). Figs 3-5 confirm this for the central kpc. We find that such variations are driven by changes in SFE and the colddense gas reservoir - in line with prior observational work focused on how SFE and available fuel in galaxies regulate their location within (and departure from) the global SFMS (e.g. Saintonge et al. 2017; Piotrowska et al. 2020; Ellison et al. 2020b). In this section, we probe deeper into the following question: what drives star formation in the central kiloparsec, efficiency or available fuel? Expressing SFR as in equation (1) facilitates this. Specifically, it allows us to write SFR enhancement as follows:

$\frac{\mathrm{SFR}}{\mathrm{SFR}_{\text {iso }}}=\frac{\mathrm{SFE}}{\mathrm{SFE}_{\text {iso }}} \times \frac{M_{\text {cold-dense }}}{M_{\text {cold-dense, iso }}}$.

Namely, in order to enhance SFR, either both SFE and colddense gas mass are enhanced simultaneously, or the enhancement of one supersedes the suppression of the other. Similarly, in order to suppress SFR, either both SFE and cold-dense gas mass are suppressed simultaneously, or the suppression of one supersedes the enhancement of the other. To describe which factor on the right-hand side of equation (3) dominates in driving their product away from unity, we adopt the following terminology:

In the enhanced-SFR regime:

(i) Efficiency-driven: $\mathrm{SFE} / \mathrm{SFE}_{\text {iso }}>M_{\text {cold-dense }} / M_{\text {cold-dense, iso }}$.

(ii) Fuel-driven: $\quad \mathrm{SFE} / \mathrm{SFE}_{\text {iso }}<M_{\text {cold-dense }} / M_{\text {cold-dense, iso }}$.

In the suppressed-SFR regime:

(i) Fuel-driven: $\quad \mathrm{SFE} / \mathrm{SFE}_{\text {iso }}>M_{\text {cold-dense }} / M_{\text {cold-dense, iso }}$

(ii) Efficiency-driven: $\mathrm{SFE} / \mathrm{SFE}_{\text {iso }}<M_{\text {cold-dense }} / M_{\text {cold-dense, iso }}$. 

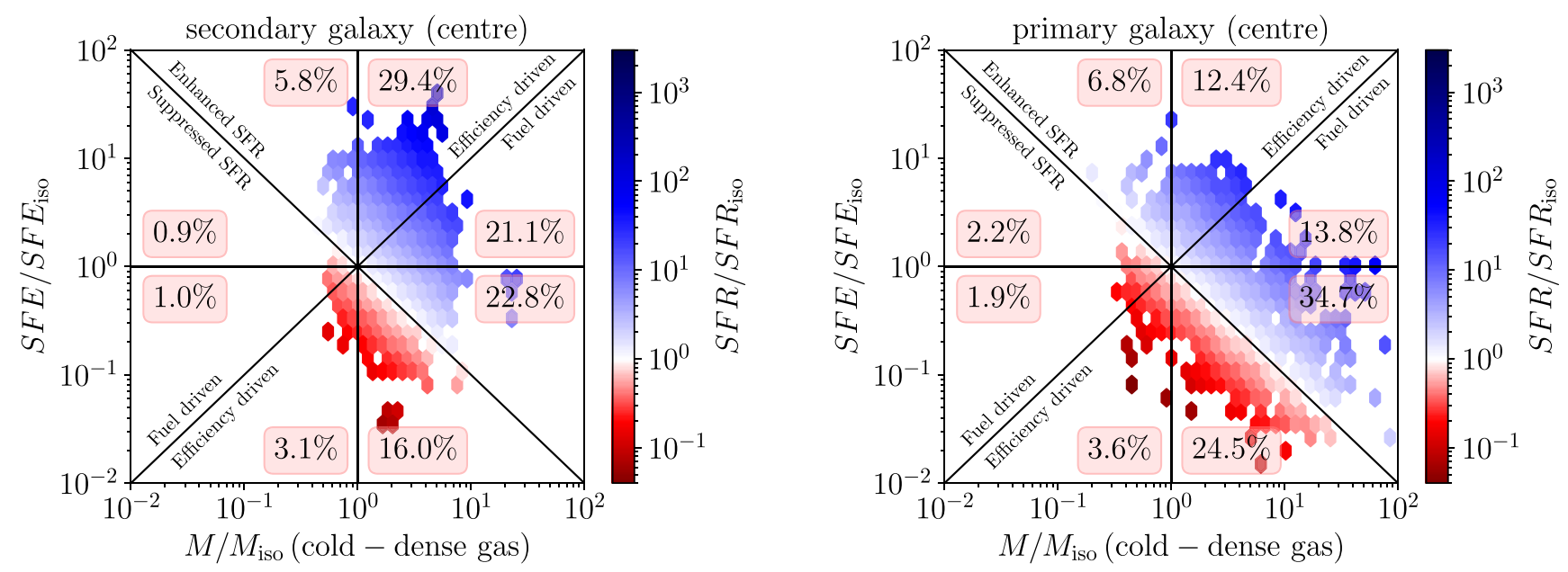

Figure 9. Dependence of central SFR enhancement on central SFE and cold-gas mass enhancements. Left (right-hand) panels: secondary (primary) galaxy. Galaxy-pair periods only. Central kiloparsec only. The 2D histogram is colour coded by central SFR enhancement, split into enhanced (blue hexagons) and suppressed values (red hexagons) - also indicated by the diagonal line with negative slope. The vertical (horizontal) line splits central cold-dense gas mass (central SFE) enhancement into enhanced and suppressed sub-samples. The diagonal line with positive slope splits the sample into situations where the dominant

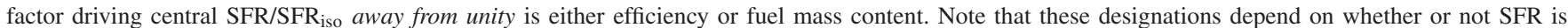
enhanced or suppressed. These four lines split each panel into eight triangular regions. Sample percentage in each triangular region is indicated.

Fig. 9 displays a 2D histogram of central SFE enhancement versus central cold-dense gas mass enhancement, colour coded by central SFR enhancement. I.e. the vertical and horizontal axes correspond to the two factors in equation (3), and the colour bar displays their product - hence the utility of the format adopted in equations (1) and (3). We include data from the entire merger suite (galaxy-pair periods only), focusing exclusively on the central kiloparsec. The left-hand panel represents the secondary galaxy, while the right-hand panel shows the primary. The horizontal and vertical lines at unity split the sample into objects with enhanced versus suppressed central SFE, and enhanced versus suppressed central cold-dense gas mass, respectively. The diagonal line with negative slope separates values with enhanced central SFR (blue hexagons) from those with suppressed central SFR (red hexagons). The diagonal line with positive slope splits the sample into efficiency-driven versus fuel-driven categories. This definition differs for SFR-enhanced and SFR-suppressed cases - i.e. it flips across the accompanying negatively sloped diagonal line (see above definitions). These four lines segregate our data into eight triangular regions. Percent contribution per triangular region is indicated.

Overall, the great majority of data points in our sample (i.e. the majority of times during the galaxy-pair period across our entire merger suite) experience enhanced central SFR: 79 per cent for the secondary galaxy and 68 per cent for the primary. Namely, not only is the average magnitude of central SFR enhancement larger in the secondary-galaxy population (by factors of 160 per cent and 70 per cent for the secondary and primary, respectively, - see Table 2 and Fig. 5), but the frequency of systems with enhanced central SFR is higher as well. Similarly, enhanced cold-dense gas content in the centre is a generic feature in our simulations: 90 per cent of cases for the secondary and 85 per cent for the primary (with average enhancement values of 130 per cent and 220 per cent). However, despite this almost ubiquitous enhancement in available cold-dense fuel within the innermost kiloparsec, central SFR is not always enhanced (e.g. red hexagons to the right of the vertical line - corresponding to 16 per cent and 24.5 per cent of cases for the secondary and primary, respectively).
For the secondary galaxy (Fig. 9, left-hand panel), 79 per cent of our sample exhibits enhanced central SFR. Within this subsample, 71 per cent have enhanced central SFE, and 92 per cent have enhanced central cold-dense gas mass. This sub-sample is almost evenly split between fuel driven and efficiency driven systems, with 55 per cent in the former category. We note that systems with the most extreme central SFR enhancements (darkest-blue hexagons) are efficiency driven (and accompanied by enhanced central colddense gas content). In the central SFR-suppressed sub-sample (the remaining 21 per cent of the entire sample), 96 per cent have suppressed central SFE, and 91 per cent occupy the efficiencydriven regime. The most extreme central SFR deficits (darkest-red hexagons) are caused by strongly suppressed central SFE, despite the presence of an abundant cold-dense gas reservoir in the centre.

For the primary galaxy (Fig. 9, right-hand panel), a smaller fraction (68 per cent) exhibit central SFR enhancement. Within this sub-sample, only 49 per cent have enhanced central SFE, and 90 per cent have enhanced cold-dense gas mass in the centre. This sub-sample has a larger fraction of fuel driven systems (71 per cent) than their secondary galaxy counterparts (only 55 per cent). Also, unlike their secondary companions, the most extreme central-SFR enhancements here (darkest-blue hexagons) are fuel driven (with central-SFE enhancement near unity). In the central SFR-suppressed regime (32 per cent of the full sample), 93 per cent of our galaxies have suppressed central SFE, and 87 per cent are in the efficiencydriven regime. As in the secondary galaxy case, the most extreme central-SFR deficits (darkest-red hexagons) occur in the efficiencydriven regime - but unlike the secondary case, these systems also contain low levels of available cold-dense gas within the inner kiloparsec.

For both galaxies, the majority of central SFR-enhanced systems are fuel driven (55 per cent and 71 per cent), and the majority of central SFR-suppressed systems are efficiency driven (91 per cent and 97 per cent) - i.e. central SFE is more strongly suppressed than their central cold-dense gas content. Also note that central SFE enhancement is more common in the secondary galaxy (57 per cent versus 35 per cent in the primary), and reaches higher levels than 
the primary (126 per cent versus 11 per cent at the upper $1 \sigma$ level - Table 2 and Fig. 5). Although central cold-dense gas mass enhancement is slightly less frequent in the primary ( 85 per cent versus 89 per cent in the secondary), it reaches higher levels than the secondary (470 per cent versus 230 per cent at the upper $1 \sigma$ level). This is manifested by an overall shift downwards and to-theright (lower efficiencies and higher cold-dense gas masses) in the 2D distribution (left versus right-hand panels in Fig. 9). In sum, this population shift explains why, even though the primary galaxy has a healthier central cold-dense gas reservoir, the secondary galaxy is more efficient at making stars in the centre (Figs 3-5).

Observations using the ALMaQUEST survey suggests that central starbursts are primarily driven by enhancements in central SFE (Ellison et al. 2020a). Here we find that, for the secondary galaxy, systems with the highest central SFR enhancements are efficiency driven - but this is not necessarily true for the primary galaxy. Note also that the central region of the secondary galaxy achieves higher central SFR enhancements relative to its primary counterpart, and has higher central SFE levels (darkest-blue hexagons in the triangular region with the '29.4 per cent' label). In other words, according to our simulations, the centres of secondary galaxies are more likely to achieve starburst status. It would be interesting to check if the three starbursts with signs of merger features in the Ellison et al. (2020a) sample are secondary companions in a galaxy pair. For galaxies not classified as starbursts, Ellison et al. (2020b) find that enhanced central SFR is more likely to be driven by high levels of $\mathrm{H}_{2}$ gas in the inner regions (see also Piotrowska et al. 2020; Bluck et al. 2020). The large fraction of fuel-driven cases with mild SFR enhancement in the centre (lighest-blue hexagons in both panels) are in line with these observations. Lastly, Thorp et al. (in preparation) report that the majority of their SFR enhanced post-mergers are SFE driven, while the majority of the SR-suppressed post-mergers are fuel-driven. Testing this result against our simulations in the postcoalescence regime is the subject of future work.

Beyond the near universal trends we describe above, there exist a few cases with enhanced central SFR and suppressed cold-dense gas mass in the centre (blue-hexagons to the left of the vertical line, corresponding to 7 per cent and 10 per cent for the secondary and primary samples, respectively) - in agreement with merging galaxies existing in nature (e.g. Arp 240, He et al. 2020). At the other extreme, a substantial fraction of our sample experiences enhancements in central SFR and cold-dense gas mass, but with suppressed central SFE (29 per cent and 51 per cent for the secondary and primary cases). Examples of systems following this trend also exist in nature, including the two nuclei of the famous Antennae galaxies (NGC 4038/39, Bemis \& Wilson 2019). It would be interesting to verify if simulations tailored to model this specific system (e.g. Renaud et al. 2014, 2015) reproduce this central behaviour.

The fact that the central kiloparsec of the primary galaxy frequently experiences low SFE levels, despite experiencing huge boosts in cold-dense gas fuel, is intriguing. This is particularly true between 0.5 and 1.5 Gyr after first pericentric passage (Figs 3 and 4). Concretely, in those situations, stellar feedback (and possibly other dynamical processes) prevents our cold-dense gas (at $n>10 \mathrm{~cm}^{-3}$ and $T<$ $300 \mathrm{~K}$ ) from reaching (or maintaining) the thresholds required for star formation (in our model, self-gravitating with $n>1000 \mathrm{~cm}^{-3}$ ). Indeed, Moreno et al. (2019) show that, in our suite of galaxymerger simulations, under $\sim 0.15$ per cent of our cold-dense gas budget achieves SF status at any given time. Such a small fraction is a reflection of the dynamic and turbulent nature of the ISM at small scales, wherein gas undergoes a cycle of collapse, star formation, and cloud dispersal - governed by feedback and possibly other dynamical processes (see e.g. Torrey et al. 2017; Semenov et al. 2017; Semenov, Kravtsov \& Gnedin 2018; Orr, Hayward \& Hopkins 2019). Detailed understanding of which processes govern low SFE levels in the presence of abundant cold-dense gas in our merger simulations is beyond the scope of this paper. Nevertheless, we highlight a wellknown (non-merging) system that exhibits this behaviour: our own Milky Way. Namely, the Central Molecular Zone (CMZ; Ferrière, Gillard \& Jean 2007; Ginsburg et al. 2016) experiences low levels of star-formation activity, despite the copious presence of moleculargas fuel (e.g. Barnes et al. 2017). Such low SFE levels are likely driven by turbulent pressure (Krumholz \& Kruijssen 2015). Jeffreson et al. (2018) suggest that between $\sim 120$ and $500 \mathrm{pc}$, galactic shear dissipates clouds - while epicyclic perturbations incite tidally driven collapse along a stream at $100 \mathrm{pc}$ from the centre. Using FIRE-2 physics, Orr et. al. (in preparation) find that their simulated galaxies reproduce the properties of the $\mathrm{CMZ}$ at some point in their evolution, but only in situations where asymmetric and bursty galactic cores are produced.

\section{SUMMARY}

We use an extensive suite of parsec-scale galaxy-merger simulations (Moreno et al. 2019) to track the spatial structure and evolution of star formation and the ISM in interacting galaxies. These simulations employ the 'FIRE-2' physics model, which is capable of capturing the multiphase structure of the ISM (Hopkins et al. 2018). In this paper, we focus on major mergers (stellar mass ratio $=2.5: 1$ ) in the galaxy-pair period, between first and second pericentric passage, with distance greater $20 \mathrm{kpc}$. We point the reader to Section 2 for relevant terminology (boldface italics) and summarize our main results below.

\section{(1) Evolution in the central kiloparsec and the outskirts.}

(a) Both galaxies experience strong central mass boosts in cool/cold-dense gas and new stars during the interaction.

(b) Despite the presence of a healthy reservoir of cold-dense gas, nuclear star formation in the primary galaxy is weak. This is caused by low SFE levels.

(c) Suppression of star formation is not statistically significant in the 1-10 kpc region - although suppression exists for a small range of radii within that region, and its strength depends on orbital geometry and time of observation after the first encounter.

\section{(2) Radial structure and evolution.}

(a) The radial extent of mass enhancement in new stars and cool/cold-dense gas is more centrally concentrated in the secondary galaxy than in the primary (within $\sim 2$ versus $\sim 7 \mathrm{kpc}$ ).

(b) The primary galaxy builds a healthier cold-dense gas reservoir, but this does not translate into more mass in new stars due to low SFE levels.

(c) As a function of time, cold-dense and cool gas mass enhancement at large radii grows, and the central peak shifts outwards and becomes more intense.

\section{(3) Dependence on orbital geometry.}

(a) The great majority of orbits in our galaxy-merger suite (two-thirds) display the behaviour described in item (2) above.

(b) A small sub-sample (one-fifth - mostly near-retrograde orbits with large separation at first pericentre) exhibit gentler changes to radial structure during the interaction. 
(c) A much smaller subset (one-eight - mostly near-polar orbits with small separation at first pericentre) experience vigorous changes in radial structure, including deep baryonic suppression at intermediate radii for the secondary galaxy, and more intense enhancement in that region for the primary.

\section{(4) Connections between global SFR and radial structure.}

(a) Globally enhanced SFs experience strong SFR enhancement in the inner region, driven by similar enhancements in nuclear SFE and available cold-dense ISM fuel.

(b) When the primary belongs to the globally suppressed SF population, SFR at small radii is suppressed due to decrements in nuclear SFE, without significant changes in available colddense ISM fuel.

\section{(5) Star formation in the central kiloparsec.}

(a) Both secondary and primary galaxies experience enhancements in central SFR (79 per cent and 68 per cent), and available cold-dense gas mass in the centre ( 89 per cent and 85 per cent).

(b) Central SFE is enhanced in the majority of cases for the secondary galaxy (57 per cent), and suppressed in the primary (67 per cent).

(c) In most cases, central SFR enhancement in both galaxies is fuel driven (55 per cent and 71 per cent), while central SFR suppression is efficiency driven (91 per cent and 97 per cent).

Our results advocate for a new class of IFU surveys i.e. either immense efforts with substantially larger galaxy samples, such as HECTOR (Lawrence et al. 2012); and/or programmes focused chiefly on galaxy mergers, such as the Snapshot Optical Spectroscopic Imaging of Mergers and Pairs for Legacy Exploration (SOSIMPLE, PI: B. Husemann). Such initiatives must also be coupled with multiwavelength follow-up campaigns (e.g. EDGECALIFA and ALMaQUEST) capable of capturing large samples of interacting galaxies at various stages, orbital geometries, locations relative to the global SFR $-M_{\star}$ relation; with representative ISM content. For direct comparison with our numerical predictions, it is imperative to measure the radial structure of interacting galaxies in relation to carefully matched non-interacting controls, and to ascribe secondary versus primary galaxy status in these targets.

Our work focuses exclusively on major galaxy interactions, meant to represent typical galaxy pairs in the local Universe. In future work, we plan to explore the effect of varying the mass ratio (Cox et al. 2008; Lotz et al. 2010), and will extend our analysis into the post-coalescence period (Thorp et al. 2019; Hani et al. 2020b; Peschken, Łokas \& Athanassoula 2020). We also intend to explore the region outside our two 10-kpc spheres, and evaluate the role of tidal compression in driving extended star formation (Renaud et al. 2014, 2015). These investigations can also be expanded into (1) the dwarf regime, where gas content and SFE might be substantially different (Stierwalt et al. 2015; Pearson et al. 2016; Kado-Fong et al. 2020; Martin et al. 2020); (2) the high-redshift regime, where modifications to ISM content and structure (Bournaud et al. 2011; Fensch et al. 2017; Calabrò et al. 2019) are accompanied by an increased frequency of merging (Bluck et al. 2009, 2012; LópezSanjuan et al. 2009; López-Sanjuan et al. 2012, 2013; RodriguezGomez et al. 2015; Mantha et al. 2018; Duncan et al. 2019); (3) minor mergers, which are expected to be more frequent (Villalobos \& Helmi 2008, 2009; Qu et al. 2011; Kaviraj 2014; Martin et al. 2018); and (4) the massive regime, where mixed and dry encounters tend to appear (Lin et al. 2008; Stewart et al. 2009; Lin et al. 2010) - and where
AGN feedback and environmental quenching processes are likely to collaborate in concert with tidal interactions, and assist galaxies in achieving their retirement into the passive sequence (Bluck et al. 2014, 2020).

\section{ACKNOWLEDGEMENTS}

We thank the anonymous reviewer for their thorough and insightful comments, which were shared with us on a heroically reasonable time-scale in the middle of the Covid-19 pandemic. Their suggestions certainly improved the quality of this manuscript. The computations in this paper were run on the Odyssey cluster supported by the FAS Division of Science, Research Computing Group at Harvard University. Support for JM is provided by the National Science Foundation (NSF, Award Number 1516374), and by the Harvard Institute for Theory and Computation, through their Visiting Scholars Program. DRP and SLE gratefully acknowledge NSERC for Discovery Grants which helped to fund this research. CB acknowledges the support of a National Sciences and Engineering Research Council of Canada (NSERC) Graduate Scholarship. AB acknowledges European Research Council (ERC) Advanced Grant 695671 'Quench' and support from the STFC. MHH acknowledges the receipt of a Vanier Canada Graduate Scholarship. The Flatiron Institute is supported by the Simons Foundation. Support for PFH is provided by NSF Collaborative Research Grants 1715847 and 1911233, NSF CAREER grant 1455342, National Aeronautics Space Agency (NASA) grants 80NSSC18K0562, <0:funding-source 3: href="http://dx.doi.org/10.13039/100006196" $\rangle$ Jet Propulsion Labor atory (JPL) $\langle/ 0$ :funding-source $\rangle 1589742$. We honour the invaluable labour of the maintenance and clerical staff at our institutions whose contributions make our scientific discoveries a reality - and urge institutions everywhere to protect these essential workers from the worldwide economic contraction caused by the pandemic. This research was conducted on Tongva-Gabrielino Indigenous land.

\section{DATA AVAILABILITY}

The data underlying this article will be shared on reasonable request to the corresponding author.

\section{REFERENCES}

Abazajian K. N. et al., 2009, ApJS, 182, 543

An S.-H., Kim J., Moon J.-S., Yoon S.-J., 2019, ApJ, 887, 59

Anglés-Alcázar D., Faucher-Giguère C.-A., Quataert E., Hopkins P. F. Feldmann R., Torrey P., Wetzel A., Kereš D., 2017, MNRAS, 472, L109 Anglés-Alcázar D. et al., 2020, preprint (arXiv:2008.12303)

Appleby S., Davé R., Kraljic K., Anglés-Alcázar D., Narayanan D., 2020, MNRAS, 494, 6053

Armus L. et al., 2009, PASP, 121, 559

Arp H., 1966, ApJS, 14, 1

Barnes J. E., Hernquist L. E., 1991, ApJ, 370, L65

Barnes J. E., Hernquist L., 1996, ApJ, 471, 115

Barnes A. T., Longmore S. N., Battersby C., Bally J., Kruijssen J. M. D., Henshaw J. D., Walker D. L., 2017, MNRAS, 469, 2263

Barrera-Ballesteros J. K. et al., 2015a, A\&A, 579, A45

Barrera-Ballesteros J. K. et al., 2015b, A\&A, 582, A21

Barton E. J., Geller M. J., Kenyon S. J., 2000, ApJ, 530, 660

Bekki K., Couch W. J., 2011, MNRAS, 415, 1783

Belfiore F. et al., 2018, MNRAS, 477, 3014

Bemis A., Wilson C. D., 2019, AJ, 157, 131

Benson A. J., 2012, New Astron., 17, 175

Bloom J. V. et al., 2017, MNRAS, 465, 123 
Bluck A. F. L., Conselice C. J., Bouwens R. J., Daddi E., Dickinson M., Papovich C., Yan H., 2009, MNRAS, 394, L51

Bluck A. F. L., Conselice C. J., Buitrago F., Grützbauch R., Hoyos C., Mortlock A., Bauer A. E., 2012, ApJ, 747, 34

Bluck A. F. L., Mendel J. T., Ellison S. L., Moreno J., Simard L., Patton D. R., Starkenburg E., 2014, MNRAS, 441, 599

Bluck A. F. L., Maiolino R., Sánchez S. F., Ellison S. L., Thorp M. D., Piotrowska J. M., Teimoorinia H., Bundy K. A., 2020, MNRAS, 492, 96

Blumenthal K. A., Barnes J. E., 2018, MNRAS, 479, 3952

Blumenthal G. R., Faber S. M., Primack J. R., Rees M. J., 1984, Nature, 311, 517

Blumenthal K. A. et al., 2020, MNRAS, 492, 2075

Bolatto A. D. et al., 2017, ApJ, 846, 159

Boselli A., Gavazzi G., 2006, PASP, 118, 517

Bournaud F., Duc P.-A., Amram P., Combes F., Gach J.-L., 2004, A\&A, 425, 813

Bournaud F. et al., 2011, ApJ, 730, 4

Bower R. G., Benson A. J., Malbon R., Helly J. C., Frenk C. S., Baugh C. M., Cole S., Lacey C. G., 2006, MNRAS, 370, 645

Brinchmann J., Charlot S., White S. D. M., Tremonti C., Kauffmann G., Heckman T., Brinkmann J., 2004, MNRAS, 351, 1151

Brownson S., Belfiore F., Maiolino R., Lin L., Carniani S., 2020, MNRAS, 498, L66

Bundy K. et al., 2015, ApJ, 798, 7

Bustamante S., Sparre M., Springel V., Grand R. J. J., 2018, MNRAS, 479, 3381

Calabrò A. et al., 2019, A\&A, 632, A98

Callegari S., Mayer L., Kazantzidis S., Colpi M., Governato F., Quinn T., Wadsley J., 2009, ApJ, 696, L89

Canalizo G., Stockton A., 2001, ApJ, 555, 719

Cano-Díaz M. et al., 2016, ApJ, 821, L26

Capelo P. R., Volonteri M., Dotti M., Bellovary J. M., Mayer L., Governato F., 2015, MNRAS, 447, 2123

Capelo P. R., Dotti M., Volonteri M., Mayer L., Bellovary J. M., Shen S., 2017, MNRAS, 469, 4437

Cappellari M., 2013, ApJ, 778, L2

Cavanagh M. K., Bekki K., 2020, A\&A, 641, A77

Chown R., Li C., Athanassoula E., Li N., Wilson C. D., Lin L., Mo H., Parker L. C., Xiao T., 2020, MNRAS, 484, 5192

Cibinel A. et al., 2019, MNRAS, 485, 5631

Cole S., Lacey C. G., Baugh C. M., Frenk C. S., 2000, MNRAS, 319, 168

Combes F., Prugniel P., Rampazzo R., Sulentic J. W., 1994, A\&A, 281, 725

Cox T. J., Jonsson P., Primack J. R., Somerville R. S., 2006, MNRAS, 373, 1013

Cox T. J., Jonsson P., Somerville R. S., Primack J. R., Dekel A., 2008, MNRAS, 384, 386

Croom S. M. et al., 2012, MNRAS, 421, 872

Croton D. J. et al., 2006, MNRAS, 365, 11

Davé R., Anglés-Alcázar D., Narayanan D., Li Q., Rafieferantsoa M. H., Appleby S., 2019, MNRAS, 486, 2827

Dekel A., Burkert A., 2014, MNRAS, 438, 1870

De Lucia G., Blaizot J., 2007, MNRAS, 375, 2

Díaz-García S., Knapen J. H., 2020, A\&A, 635, A197

Di Matteo T., Springel V., Hernquist L., 2005, Nature, 433, 604

Di Matteo P., Combes F., Melchior A.-L., Semelin B., 2007, A\&A, 468, 61

Di Matteo P., Bournaud F., Martig M., Combes F., Melchior A.-L., Semelin B., 2008, A\&A, 492, 31

Duncan K. et al., 2019, ApJ, 876, 110

D’Onghia E., Vogelsberger M., Faucher-Giguere C.-A., Hernquist L., 2010, ApJ, 725, 353

Elbaz D. et al., 2007, A\&A, 468, 33

Ellison S. L., Patton D. R., Simard L., McConnachie A. W., 2008, AJ, 135, 1877

Ellison S. L., Patton D. R., Mendel J. T., Scudder J. M., 2011, MNRAS, 418, 2043

Ellison S. L., Mendel J. T., Scudder J. M., Patton D. R., Palmer M. J. D., 2013, MNRAS, 430, 3128
Ellison S. L., Sánchez S. F., Ibarra-Medel H., Antonio B., Mendel J. T., Barrera-Ballesteros J., 2018a, MNRAS, 474, 2039

Ellison S. L., Catinella B., Cortese L., 2018b, MNRAS, 478, 3447

Ellison S. L., Viswanathan A., Patton D. R., Bottrell C., McConnachie A. W., Gwyn S., Cuillandre J.-C., 2019, MNRAS, 487, 2491

Ellison S. L., Thorp M. D., Pan H.-A., Lin L., Scudder J. M., Bluck A. F. L., Sánchez S. F., Sargent M., 2020a, MNRAS, 492, 6027

Ellison S. L. et al., 2020b, MNRAS, 493, L39

Emami N., Siana B., Weisz D. R., Johnson B. D., Ma X., El-Badry K., 2019, ApJ, 881, 71

Feng S., Shen S.-Y., Yuan F.-T., Riffel R. A., Pan K., 2020, ApJ, 892, L20

Fensch J. et al., 2017, MNRAS, 465, 1934

Ferrière K., Gillard W., Jean P., 2007, A\&A, 467, 611

Flores Velázquez J. A. et al., 2020, preprint (arXiv:2008.08582)

Ginsburg A. et al., 2016, A\&A, 586, A50

González Delgado R. M. et al., 2016, A\&A, 590, A44

Guo Q., White S., Angulo R. E., Henriques B., Lemson G., Boylan-Kolchin M., Thomas P., Short C., 2013, MNRAS, 428, 1351

Hani M. H., Sparre M., Ellison S. L., Torrey P., Vogelsberger M., 2018, MNRAS, 475,1160

Hani M. H., Hayward C. C., Orr M. E., Ellison S. L., Torrey P., Murray N., Wetzel A., Faucher-Giguère C.-A., 2020a, MNRAS, 493, L87

Hani M. H., Gosain H., Ellison S. L., Patton D. R., Torrey P., 2020b, MNRAS, 493, 3716

Hayward C. C., Torrey P., Springel V., Hernquist L., Vogelsberger M., 2014, MNRAS, 442, 1992

He H., Wilson C. D., Sliwa K., Iono D., Saito T., 2020, MNRAS, 496, 5243

Henriques B., Maraston C., Monaco P., Fontanot F., Menci N., De Lucia G., Tonini C., 2011, MNRAS, 415, 3571

Hernquist L., 1989, Nature, 340, 687

Holmberg E., 1940, ApJ, 92, 200

Holmberg E., 1941, ApJ, 94, 385

Hopkins P. F., 2017, preprint (arXiv:1712.01294)

Hopkins P. F., Hernquist L., Martini P., Cox T. J., Robertson B., Di Matteo T., Springel V., 2005, ApJ, 625, L71

Hopkins P. F., Hernquist L., Cox T. J., Kereš D., 2008, ApJS, 175, 356

Hopkins P. F., Cox T. J., Hernquist L., Narayanan D., Hayward C. C., Murray N., 2013a, MNRAS, 430, 1901

Hopkins P. F., Narayanan D., Murray N., 2013b, MNRAS, 432, 2647

Hopkins P. F., Kereš D., Oñorbe J., Faucher-Giguère C.-A., Quataert E., Murray N., Bullock J. S., 2014, MNRAS, 445, 581

Hopkins P. F., Torrey P., Faucher-Giguère C.-A., Quataert E., Murray N., 2016, MNRAS, 458, 816

Hopkins P. F. et al., 2018, MNRAS, 480, 800

Hsieh B. C. et al., 2017, ApJ, 851, L24

Hung C.-L. et al., 2013, ApJ, 778, 129

Hung C.-L., Hayward C. C., Smith H. A., Ashby M. L. N., Lanz L., MartínezGalarza J. R., Sanders D. B., Zezas A., 2016, ApJ, 816, 99

Jeffreson S. M. R., Kruijssen J. M. D., Krumholz M. R., Longmore S. N., 2018, MNRAS, 478, 3380

Jogee S. et al., 2009, ApJ, 697, 1971

Joshi G. D., Pillepich A., Nelson D., Marinacci F., Springel V., RodriguezGomez V., Vogelsberger M., Hernquist L., 2020, MNRAS, 496, 2673

Just D. W., Zaritsky D., Sand D. J., Desai V., Rudnick G., 2010, ApJ, 711, 192

Kado-Fong E., Greene J. E., Greco J. P., Beaton R., Goulding A. D., Johnson S. D., Komiyama Y., 2020, AJ, 159, 103

Karl S. J., Naab T., Johansson P. H., Kotarba H., Boily C. M., Renaud F., Theis C., 2010, ApJ, 715, L88

Karl S. J., Fall S. M., Naab T., 2011, ApJ, 734, 11

Karl S. J., Lunttila T., Naab T., Johansson P. H., Klaas U., Juvela M., 2013, MNRAS, 434, 696

Kaviraj S., 2014, MNRAS, 440, 2944

Kennicutt R. C., Jr, 1998, ARA\&A, 36, 189

Kim J.-h., Wise J. H., Abel T., 2009, ApJ, 694, L123

Knapen J. H., James P. A., 2009, ApJ, 698, 1437

Krumholz M. R., Gnedin N. Y., 2011, ApJ, 729, 36

Krumholz M. R., Kruijssen J. M. D., 2015, MNRAS, 453, 739 
Lagos C. d. P., Tobar R. J., Robotham A. S. G., Obreschkow D., Mitchell P. D., Power C., Elahi P. J., 2018, MNRAS, 481, 3573

Lagos C. d. P. et al., 2019, MNRAS, 489, 4196

Lambas D. G., Tissera P. B., Alonso M. S., Coldwell G., 2003, MNRAS, 346, 1189

Larson R. B., Tinsley B. M., 1978, ApJ, 219, 46

Lawrence J. et al., 2012, in McLean I. S., Ramsay S. K., Takami H., eds, Proc. SPIE Conf. Ser. Vol. 8446, Ground-based and Airborne Instrumentation for Astronomy IV. SPIE, Bellingham, p. 844653

Leitherer C. et al., 1999, ApJS, 123, 3

L'Huillier B., Park C., Kim J., 2015, MNRAS, 451, 527

Li S.-l. et al., 2019, preprint (arXiv:1912.04522)

Lin L. et al., 2008, ApJ, 681, 232

Lin L. et al., 2010, ApJ, 718, 1158

Lin L. et al., 2019, ApJ, 884, L33

Lisenfeld U., Xu C. K., Gao Y., Domingue D. L., Cao C., Yun M. S., Zuo P., 2019, A\&A, 627, A107

Łokas E. L., 2019, A\&A, 624, A37

Łokas E. L., Ebrová I., del Pino A., Sybilska A., Athanassoula E., Semczuk M., Gajda G., Fouquet S., 2016, ApJ, 826, 227

López-Sanjuan C., Balcells M., Pérez-González P. G., Barro G., García-Dabó C. E., Gallego J., Zamorano J., 2009, A\&A, 501, 505

López-Sanjuan C. et al., 2012, A\&A, 548, A7

López-Sanjuan C. et al., 2013, A\&A, 553, A78

Lotz J. M., Jonsson P., Cox T. J., Primack J. R., 2010, MNRAS, 404, 575

Mantha K. B. et al., 2018, MNRAS, 475, 1549

Martig M., Bournaud F., 2008, MNRAS, 385, L38

Martin G., Kaviraj S., Devriendt J. E. G., Dubois Y., Pichon C., 2018, MNRAS, 480, 2266

Martin G. et al., 2020, preprint (arXiv:2007.07913)

Martínez-Galarza J. R. et al., 2016, ApJ, 817, 76

Medling A. M. et al., 2018, MNRAS, 475, 5194

Menci N., Cavaliere A., Fontana A., Giallongo E., Poli F., Vittorini V., 2004, ApJ, 604, 12

Mendel J. T., Simard L., Palmer M., Ellison S. L., Patton D. R., 2014, ApJS, 210,3

Mesa V., Duplancic F., Alonso S., Coldwell G., Lambas D. G., 2014, MNRAS, 438, 1784

Mihos J. C., Hernquist L., 1996, ApJ, 464, 641

Moore B., Katz N., Lake G., Dressler A., Oemler A., 1996, Nature, 379, 613

Moreno J., 2012, MNRAS, 419, 411

Moreno J., Bluck A. F. L., Ellison S. L., Patton D. R., Torrey P., Moster B. P., 2013, MNRAS, 436, 1765

Moreno J., Torrey P., Ellison S. L., Patton D. R., Bluck A. F. L., Bansal G., Hernquist L., 2015, MNRAS, 448, 1107

Moreno J. et al., 2019, MNRAS, 485, 1320

Morselli L., Popesso P., Cibinel A., Oesch P. A., Montes M., Atek H., Illingworth G. D., Holden B., 2019, A\&A, 626, A61

Noeske K. G., Weiner B. J., Faber S. M., et a., 2007, ApJ, 660, L43

Orr M. E. et al., 2017, ApJ, 849, L2

Orr M. E. et al., 2018, MNRAS, 478, 3653

Orr M. E., Hayward C. C., Hopkins P. F., 2019, MNRAS, 486, 4724

Pan H.-A. et al., 2018, ApJ, 868, 132

Pan H.-A. et al., 2019, ApJ, 881, 119

Patton D. R., Pritchet C. J., Yee H. K. C., Ellingson E., Carlberg R. G., 1997, ApJ, 475, 29

Patton D. R., Torrey P., Ellison S. L., Mendel J. T., Scudder J. M., 2013, MNRAS, 433, L59

Patton D. R., Qamar F. D., Ellison S. L., Bluck A. F. L., Simard L., Mendel J. T., Moreno J., Torrey P., 2016, MNRAS, 461, 2589

Patton D. R. et al., 2020, MNRAS, 494, 4969

Pearson S. et al., 2016, MNRAS, 459, 1827

Pearson W. J. et al., 2019, A\&A, 631, A51

Perez J., Michel-Dansac L., Tissera P. B., 2011, MNRAS, 417, 580

Perez M. J., Tissera P. B., Lambas D. G., Scannapieco C., 2006, A\&A, 449, 23

Peschken N., Łokas E. L., Athanassoula E., 2020, MNRAS, 493, 1375

Pettitt A. R., Wadsley J. W., 2018, MNRAS, 474, 5645
Pillepich A. et al., 2018, MNRAS, 473, 4077

Piotrowska J. M., Bluck A. F. L., Maiolino R., Concas A., Peng Y., 2020, MNRAS, 492, L6

Privon G. C., 2014, PhD thesis, University of Virginia

Puech M., Hammer F., Rodrigues M., Fouquet S., Flores H., Disseau K., 2014, MNRAS, 443, L49

Qu Y., Di Matteo P., Lehnert M. D., van Driel W., Jog C. J., 2011, A\&A, 535 , A5

Renaud F., Boily C. M., Naab T., Theis C., 2009, ApJ, 706, 67

Renaud F., Bournaud F., Kraljic K., Duc P. A., 2014, MNRAS, 442, L33

Renaud F., Bournaud F., Duc P.-A., 2015, MNRAS, 446, 2038

Renaud F., Bournaud F., Daddi E., Weiß A., 2019a, A\&A, 621, A104

Renaud F., Bournaud F., Agertz O., Kraljic K., Schinnerer E., Bolatto A., Daddi E., Hughes A., 2019b, A\&A, 625, A65

Rey M. P., Pontzen A., 2018, MNRAS, 474, 45

Rich J. A., Torrey P., Kewley L. J., Dopita M. A., Rupke D. S. N., 2012, ApJ, 753,5

Robotham A. S. G. et al., 2014, MNRAS, 444, 3986

Rodriguez-Gomez V. et al., 2015, MNRAS, 449, 49

Rodríguez Montero F., Davé R., Wild V., Anglés-Alcázar D., Narayanan D., 2019, MNRAS, 490, 2139

Roth N., Pontzen A., Peiris H. V., 2016, MNRAS, 455, 974

Rupke D. S. N., Kewley L. J., Chien L.-H., 2010, ApJ, 723, 1255

Sabater J., Best P. N., Argudo-Fernández M., 2013, MNRAS, 430, 638

Saintonge A. et al., 2012, ApJ, 758, 73

Saintonge A. et al., 2016, MNRAS, 462, 1749

Saintonge A. et al., 2017, ApJS, 233, 22

Salim S., Lee J. C., Ly C., Brinchmann J., Davé R., Dickinson M., Salzer J. J., Charlot S., 2014, ApJ, 797, 126

Sánchez S. F. et al., 2012, A\&A, 538, A8

Sanders D. B., Mirabel I. F., 1996, ARA\&A, 34, 749

Sanders D. B., Soifer B. T., Elias J. H., Madore B. F., Matthews K., Neugebauer G., Scoville N. Z., 1988, ApJ, 325, 74

Satyapal S., Secrest N. J., McAlpine W., Ellison S. L., Fischer J., Rosenberg J. L., 2014, ApJ, 784, 113

Schaye J. et al., 2015, MNRAS, 446, 521

Scudder J. M., Ellison S. L., Torrey P., Patton D. R., Mendel J. T., 2012, MNRAS, 426, 549

Semenov V. A., Kravtsov A. V., Gnedin N. Y., 2017, ApJ, 845, 133

Semenov V. A., Kravtsov A. V., Gnedin N. Y., 2018, ApJ, 861, 4

Silva A. et al., 2018, ApJ, 868, 46

Sinha M., Holley-Bockelmann K., 2012, ApJ, 751, 17

Smith B. J., Campbell K., Struck C., Soria R., Swartz D., Magno M., Dunn B., Giroux M. L., 2018, AJ, 155, 81

Smith B. J., Wagstaff P., Struck C., Soria R., Dunn B., Swartz D., Giroux M. L., 2019, AJ, 158, 169

Somerville R. S., Hopkins P. F., Cox T. J., Robertson B. E., Hernquist L., 2008, MNRAS, 391, 481

Sparre M., Springel V., 2016, MNRAS, 462, 2418

Sparre M., Hayward C. C., Feldmann R., Faucher-Giguère C.-A., Muratov A. L., Kereš D., Hopkins P. F., 2017, MNRAS, 466, 88

Spindler A. et al., 2018, MNRAS, 476, 580

Springel V., Hernquist L., 2003, MNRAS, 339, 289

Stewart K. R., Bullock J. S., Barton E. J., Wechsler R. H., 2009, ApJ, 702, 1005

Stierwalt S., Besla G., Patton D., Johnson K., Kallivayalil N., Putman M., Privon G., Ross G., 2015, ApJ, 805, 2

Stopyra S., Pontzen A., Peiris H., Roth N., Rey M., 2020, preprint (arXiv: 2006.01841)

Tacchella S., Dekel A., Carollo C. M., Ceverino D., DeGraf C., Lapiner S., Mand elker N., Primack Joel R., 2016a, MNRAS, 457, 2790

Tacchella S., Dekel A., Carollo C. M., Ceverino D., DeGraf C., Lapiner S., Mand elker N., Primack J. R., 2016b, MNRAS, 458, 242

Teyssier R., Chapon D., Bournaud F., 2010, ApJ, 720, L149

Thorp M. D., Ellison S. L., Simard L., Sánchez S. F., Antonio B., 2019, MNRAS, 482, L55

Tonnesen S., Cen R., 2012, MNRAS, 425, 2313

Toomre A., Toomre J., 1972, ApJ, 178, 623 
Torrey P., Vogelsberger M., Sijacki D., Springel V., Hernquist L., 2012, MNRAS, 427, 2224

Torrey P., Hopkins P. F., Faucher-Giguère C.-A., Vogelsberger M., Quataert E., Kereš D., Murray N., 2017, MNRAS, 467, 2301

Treister E., Schawinski K., Urry C. M., Simmons B. D., 2012, ApJ, 758, L39

Villalobos Á., Helmi A., 2008, MNRAS, 391, 1806

Villalobos Á., Helmi A., 2009, MNRAS, 399, 166

Violino G., Ellison S. L., Sargent M., Coppin K. E. K., Scudder J. M., Mendel T. J., Saintonge A., 2018, MNRAS, 476, 2591

Vogelsberger M., Genel S., Sijacki D., Torrey P., Springel V., Hernquist L., 2013, MNRAS, 436, 3031

Wang E., Lilly S. J., Pezzulli G., Matthee J., 2019, ApJ, 877, 132

Whitaker K. E., van Dokkum P. G., Brammer G., Franx M., 2012, ApJ, 754, L29

White S. D. M., Frenk C. S., 1991, ApJ, 379, 52

White S. D. M., Rees M. J., 1978, MNRAS, 183, 341

Wilkinson C. L., Pimbblet K. A., Stott J. P., Few C. G., Gibson B. K., 2018, MNRAS, 479, 758
Willett K. W. et al., 2015, MNRAS, 449, 820

Woo J., Ellison S. L., 2019, MNRAS, 487, 1927

Yamashita T. et al., 2017, ApJ, 844, 96

Zolotov A. et al., 2015, MNRAS, 450, 2327

\section{SUPPORTING INFORMATION}

Supplementary data are available at MNRAS online.

Figure S1. Terminology and three illustrative examples.

Please note: Oxford University Press is not responsible for the content or functionality of any supporting material supplied by the authors. Any queries (other than missing material) should be directed to the corresponding author for the article.

This paper has been typeset from a $\mathrm{T}_{\mathrm{E}} \mathrm{X} / \mathrm{L} \mathrm{AT} \mathrm{E}$ file prepared by the author. 